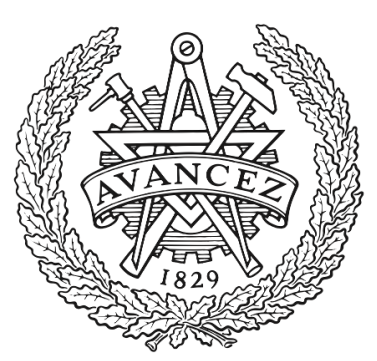

CHALMERS

UNIVERSITY OF TECHNOLOGY

\title{
Resolving the ISM at the Peak of Cosmic Star Formation with ALMA: The Distribution of CO and Dust Continuum in $\mathrm{z} \square 2.5$ Submillimeter Galaxies
}

Downloaded from: https://research.chalmers.se, 2023-04-26 10:16 UTC

Citation for the original published paper (version of record):

Rivera, G., Hodge, J., Smail, I. et al (2018). Resolving the ISM at the Peak of Cosmic Star Formation with ALMA: The Distribution of CO and Dust Continuum in z $\square 2.5$ Submillimeter Galaxies. Astrophysical Journal, 863(1). http://dx.doi.org/10.3847/1538-4357/aacffa

N.B. When citing this work, cite the original published paper. 


\title{
Resolving the ISM at the Peak of Cosmic Star Formation with ALMA: The Distribution of $\mathrm{CO}$ and Dust Continuum in $z \sim 2.5$ Submillimeter Galaxies
}

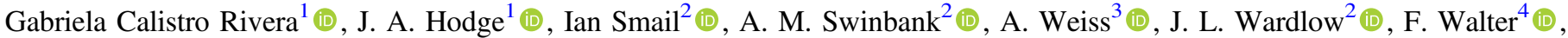 \\ M. Rybak ${ }^{1}$, Chian-Chou Chen ${ }^{5}$ (D), W. N. Brandt ${ }^{6,7,8}$ (D) , K. Coppin ${ }^{9}$ (D), E. da Cunha ${ }^{10}$, H. Dannerbauer ${ }^{11,12}$ (iD), T. R. Greve ${ }^{13}$ (iD), \\ A. $\operatorname{Karim}^{14}$ (D) K. K. Knudsen ${ }^{15}$ (D), E. Schinnerer ${ }^{4}$ (D) J. M. Simpson ${ }^{16}$ (D) B. Venemans ${ }^{4}$ (D), and P. P. van der Werf ${ }^{1}$ \\ ${ }^{1}$ Leiden Observatory, Leiden University, P.O. Box 9513, 2300 RA Leiden, The Netherlands; calistro@strw.leidenuniv.nl \\ ${ }^{2}$ Centre for Extragalactic Astronomy, Department of Physics, Durham University, South Road, Durham DH1 3LE, UK \\ ${ }^{3}$ Max-Planck-Institut für Radioastronomie, Auf dem Hügel 69, D-53121 Bonn, Germany \\ ${ }_{5}^{4}$ Max Planck Institute for Astronomy, Königstuhl 17, D-69117 Heidelberg, Germany; \\ ${ }^{5}$ European Southern Observatory, Karl Schwarzschild Strasse 2, Garching, Germany \\ ${ }^{6}$ Department of Astronomy \& Astrophysics, 525 Davey Lab, Pennsylvania State University, University Park, PA 16802, USA \\ ${ }^{7}$ Institute for Gravitation and the Cosmos, Pennsylvania State University, University Park, PA 16802, USA \\ ${ }^{8}$ Department of Physics, The Pennsylvania State University, University Park, PA 16802, USA \\ ${ }^{9}$ Centre for Astrophysics Research, University of Hertfordshire, Hatfield, AL10 9AB, UK \\ ${ }^{10}$ The Australian National University, Mt Stromlo Observatory, Cotter Rd, Weston Creek, ACT 2611, Australia \\ ${ }^{11}$ Instituto de Astrofísica de Canarias (IAC), E-38205 La Laguna, Tenerife, Spain \\ ${ }^{12}$ Universidad de La Laguna, Dpto. Astrofisica, E-38206 La Laguna, Tenerife, Spain \\ ${ }^{13}$ Department of Physics and Astronomy, University College London, Gower Street, London, WC1E 6BT, UK \\ ${ }^{14}$ Argelander-Institute of Astronomy, Bonn University, Auf dem Hügel 71, D-53121 Bonn, Germany

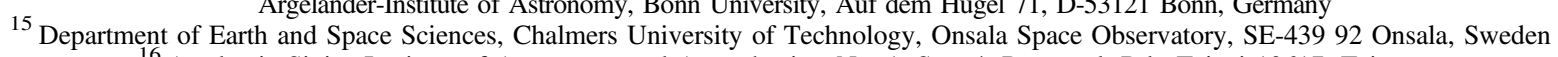 \\ ${ }^{16}$ Academia Sinica Institute of Astronomy and Astrophysics, No. 1, Sec. 4, Roosevelt Rd., Taipei 10617, Taiwan \\ Received 2018 March 2; revised 2018 June 11; accepted 2018 June 25; published 2018 August 9
}

\begin{abstract}
We use Atacama Large Millimeter Array (ALMA) observations of four submillimeter galaxies (SMGs) at $z \sim 2-3$ to investigate the spatially resolved properties of the interstellar medium (ISM) at scales of 1-5 kpc (0." $1-0$ !" 6). The velocity fields of our sources, traced by the ${ }^{12} \mathrm{CO}(J=3-2)$ emission, are consistent with disk rotation to the first order, implying average dynamical masses of $\sim 3 \times 10^{11} M_{\odot}$ within two half-light radii. Through a Bayesian approach we investigate the uncertainties inherent to dynamically constraining total gas masses. We explore the covariance between the stellar mass-to-light ratio and $\mathrm{CO}$-to- $\mathrm{H}_{2}$ conversion factor, $\alpha_{\mathrm{CO}}$, finding values of $\alpha_{\mathrm{CO}}=1.1_{-0.7}^{+0.8}$ for dark matter fractions of $15 \%$. We show that the resolved spatial distribution of the gas and dust continuum can be uncorrelated to the stellar emission, challenging energy balance assumptions in global SED fitting. Through a stacking analysis of the resolved radial profiles of the $\mathrm{CO}(3-2)$, stellar, and dust continuum emission in SMG samples, we find that the cool molecular gas emission in these sources (radii $\sim 5-14 \mathrm{kpc}$ ) is clearly more extended than the rest-frame $\sim 250 \mu \mathrm{m}$ dust continuum by a factor $>2$. We propose that assuming a constant dust-to-gas ratio, this apparent difference in sizes can be explained by temperature and optical depth gradients alone. Our results suggest that caution must be exercised when extrapolating morphological properties of dust continuum observations to conclusions about the molecular gas phase of the interstellar medium (ISM).
\end{abstract}

Key words: galaxies: ISM - galaxies: kinematics and dynamics - galaxies: structure - ISM: structure submillimeter: galaxies - submillimeter: ISM

\section{Introduction}

The process of massive galaxy assembly in the universe has been identified to peak between $1<z<3$, where the majority of the stars in the present-day galaxies formed (e.g., Madau \& Dickinson 2014). This star formation (SF) is likely to be strongly connected to the gas content and its distribution in the interstellar medium (ISM) and the efficiency with which this gas is transformed into stars (e.g., Decarli et al. 2016; Scoville et al. 2017; Tacconi et al. 2017). Gas-rich, dusty galaxies, as submillimeter galaxies (SMGs; e.g., Blain et al. 2002) are effective laboratories to characterize this star-forming ISM due to their high molecular gas content (Bothwell et al. 2013) and their bright dust continuum emission ensured by their selection. Moreover, they appear to contribute around $20 \%$ of the total SF rate density at $z \sim 2-3$ (e.g., Swinbank et al. 2014) and are thus an important tracer of the SF occurring in massive galaxies at this epoch.
The characterization of the star-forming ISM at these high redshifts is typically achieved through observations of the rotational transitions of carbon monoxide $(\mathrm{CO})$ or through deep imaging of the Rayleigh-Jeans (RJ) tail of the dust continuum emission (for reviews see, Carilli \& Walter 2013; Casey et al. 2014). However, calculations based on these ISM tracers involve a number of assumptions about the inferred gas properties. Although $\mathrm{CO}$ is the most strongly emitting molecule, it is only the second most abundant molecule in the galaxy ISM after molecular Hydrogen, $\mathrm{H}_{2}$, and a conversion factor $\left(\alpha_{\mathrm{CO}}\right)$ from the ground state $\mathrm{CO}(J=1-0)$ luminosity to $\mathrm{H}_{2}$ (e.g., Bolatto et al. 2013) is thus required to compute the total molecular content. As a result there have been numerous observational (e.g., Tacconi et al. 2008; Danielson et al. 2011; Genzel et al. 2012; Hodge et al. 2012; Bolatto et al. 2013; Bothwell et al. 2013; Accurso et al. 2017) and theoretical (e.g., Narayanan et al. 2011, 2012; Lagos et al. 2012) efforts to constrain $\alpha_{\mathrm{CO}}$ in different galaxy populations, which 
represents a significant uncertainty in total gas mass estimations.

In the local universe, the range of $\alpha_{\mathrm{CO}}$ values is observed to span a factor of $\sim 5$ and it has been shown to be a function of several galaxy properties such as gas density, temperature, and metallicity (Bolatto et al. 2013), which likely evolve with redshift.

Another approach to estimate galaxy gas masses is to use the dust continuum observations as a proxy for the ISM content at low and intermediate redshifts (e.g., Hildebrand 1983; Leroy et al. 2011; Magdis et al. 2011; Scoville et al. 2014). Recently, Scoville et al. (2016) combined molecular gas masses inferred from existing $\mathrm{CO}$ detections and dust continuum measurements in an attempt to calibrate an empirical scaling factor for using global measurements of the RJ dust continuum (probed in the submm regime) to estimate the total ISM masses. Although they find that this calibration holds for measurements over three orders of magnitude in infrared luminosity and for different populations including SMGs, this is based on significant assumptions about the properties of the dust spectral energy distribution (SED) in addition to the uncertainties on the $\alpha_{\mathrm{CO}}$ parameter assumed for the calibration. To test the validity of these assumptions, observational constraints on the physics of the high-redshift ISM are required.

Spatially unresolved high-redshift surveys of $\mathrm{CO}$ and dust continuum have begun to make progress on these tracers and their implications for the SF picture from a global perspective. Over 200 detections of $\mathrm{CO}$ line emission at high redshift have been reported $(z<2$; Carilli \& Walter 2013) from both individual sources (e.g., Genzel et al. 2012; Hodge et al. 2012; Strandet et al. 2017) and larger statistical surveys (Ivison et al. 2011; Bothwell et al. 2013; Sharon et al. 2016; Walter et al. 2016). In addition, multiple submm continuum surveys have been conducted to characterize the dust emission and population properties of SMGs (Hodge et al. 2013b; Karim et al. 2013; Simpson et al. 2017). Statistical studies (e.g., Bothwell et al. 2013; Sharon et al. 2016) have shed light on the average level of excitation for SMGs, which are observed to have large scatter at high excitation levels $J \geqslant 4$ (e.g., Narayanan \& Krumholz 2014) but behave relatively homogeneously at $J \leqslant 3$ with excitation ratios of $r_{3-1}=0.78 \pm$ 0.27 (Sharon et al. 2016). Moreover, high excitation CO transitions $(J \geqslant 4)$ have been observed to underestimate the gas masses, since they appear to be biased to trace the most compact molecular emission only (e.g., Engel et al. 2010). Finally, using ISM mass estimates based on dust continuum observations and assuming a constant dust/gas ratio (e.g., Leroy et al. 2011), Genzel et al. (2015), Scoville et al. (2017) and Tacconi et al. (2017) were able to investigate statistically the evolution of the unresolved star-forming ISM for SF galaxies across cosmic time.

At high resolution, significant progress with the characterization of the high-redshift ISM has been achieved through the observation of gravitationally lensed sources, as part of the surveys conducted by the South Pole Telescope (SPT; e.g., Greve et al. 2012; Vieira et al. 2013; Spilker et al. 2016) and Herschel (e.g., Harris et al. 2012; Wardlow et al. 2013). Lensing observations, however, are prone to be affected by differential magnification when a detailed lensing model is not available (e.g., Hezaveh et al. 2012; Serjeant 2012). Moreover, caution must be exercised when interpreting intrinsic integrated properties of the $\mathrm{CO}$ emission in strongly lensed galaxies, since these can be biased against the less magnified regions.

Only a handful of studies have characterized the ISM on subgalactic scales in unlensed high-redshift galaxies (e.g., Tacconi et al. 2008; Engel et al. 2010; Hodge et al. 2012, 2013a; Aravena et al. 2014; Decarli et al. 2016; Chen et al. 2017; Tadaki et al. 2017b), and even fewer have studied the dust continuum emission and gas observations on comparable scales. High-resolution imaging is crucial for characterizing the ISM in galaxies, since apart from providing a better morphological description of the gas or dust continuum emission, this information is key for estimating fundamental properties such as gas surface density. Moreover, through dynamical modeling of the velocity fields, one can derive dynamical mass estimates (de Blok \& Walter 2014), which reflect the total mass of baryonic and non baryonic matter contained within the region traced by the observed line emission, and thus constrains the sum of stellar, gas, and dark matter (DM) masses. When complemented with stellar mass and DM fraction assumptions, dynamical mass estimates can constrain the total mass of gas reservoirs (Tacconi et al. 2008; Hodge et al. 2012), and hence the $\alpha_{\mathrm{CO}}$ parameter.

However, estimating the mass of the other components is also complex, for example, the stellar mass of a galaxy is usually inferred via SED fitting, which is prone to induce strong degeneracies between parameters such as the SF history, dust reddening, luminosity-weighted age of the stellar populations and mass-to-light ratio, especially for highly star-forming galaxies such as SMGs (e.g., Hainline et al. 2011; Simpson et al. 2014, 2017). The DM fraction represents a large source of uncertainty, as no independent measurement of its mass is possible. While recent spectroscopic surveys have claimed DM fractions around 10\%-30\%, (Price et al. 2016; Wuyts et al. 2016; Genzel et al. 2017) revealing that star-forming galaxies at $z>2$ appear to be heavily baryon dominated, these calculations involve making similar uncertain assumptions about the gas fractions. Shedding light on the impact of these uncertainties on the $\alpha_{\mathrm{CO}}$ and gas mass estimations is thus imperative for an improved characterization of the highredshift ISM.

The collecting area and sensitivity of the Atacama Large Millimeter Array (ALMA) is transforming our view of the starforming ISM in distant galaxies. While high-resolution studies of the dust continuum emission in SMGs with ALMA have shown that this material appears to be mostly distributed in compact regions (Ikarashi et al. 2015; Simpson et al. 2015; Hodge et al. 2016; Tadaki et al. 2017a), the extended sizes revealed by the few resolved $\mathrm{CO}$ detections of luminous sources (Riechers et al. 2010; Hodge et al. 2012; Emonts et al. 2016; Chen et al. 2017; Dannerbauer et al. 2017; Ginolfi et al. 2017; Tadaki et al. 2017b) challenge the general assumptions of co-spatial interwoven dust and gas generally assumed by other models. Spatially resolved observations of $\mathrm{CO}$ and dust continuum emission for homogeneously selected samples and modeling of their interplay, e.g., through radiative transfer approximations, may help to characterize the distributions and physics of dust and gas in the high-redshift ISM.

Here, we present high-resolution imaging of the $\mathrm{CO}$ emission in four SMGs from the ALESS survey (Hodge et al. 2013b; Karim et al. 2013) at sub-arcsecond resolution. We address the following questions that remain open in ISM studies at high redshift: how is the molecular gas distributed in 
relation to the dusty ISM and the stellar populations? What implications do these distributions have for the assumptions made for the dust SEDs and dust-to-gas ratios at high redshift? How reliable are gas mass estimations? What uncertainties do stellar mass estimates and DM fraction assumptions introduce into the total gas mass estimation? The paper is structured as follows: in Section 2 we describe the ALMA data reduction and the imaging of the $\mathrm{CO}(3-2)$ maps. In Section 3 we present the analysis of the kinematic properties of the $\mathrm{CO}(3-2)$ emission in our sources and present the implications of these to total gas estimates. In Section 4 we present a statistical analysis of the distributions of gas, dust continuum and stellar emission and discuss the physical implications of these findings. Throughout the paper we adopt a $\Lambda$-CDM cosmology, consistent with the values given by the Planck Collaboration et al. (2014), with $\Omega_{\Lambda}=0.69, \Omega_{m}=$ 0.31 and $H_{0}=67.7 \mathrm{~km} \mathrm{~s}^{-1} \mathrm{Mpc}^{-1}$.

\section{Observations and Imaging}

\subsection{Target Selection ALMA Observations}

We present ALMA Cycle 2 observations of the CO emission from four SMGs, ALESS49.1, ALESS57.1, ALESS67.1, and ALESS122.1. These sources were selected from the ALESS survey (Hodge et al. 2013b; Karim et al. 2013): an ALMA Cycle 0 follow-up program of 126 sources detected in the single-dish LABOCA Extended Chandra Deep Field South (ECDFS) Submm Survey (LESS, Weiß et al. 2009). These four sources were selected as they are spectroscopically confirmed at redshifts $2.1<z<2.9$ as the result of an extensive spectroscopic follow-up campaign (Danielson et al. 2017). Three of the four sources in our sample were detected (ALESS57.1 and ALESS67.1) or marginally detected (ALESS122.1, through stacking) in the X-rays (Wang et al. 2013), using 4 Ms Chandra observations of the CDFS region (Xue et al. 2011) and $250 \mathrm{ks}$ observations in the ECDFS (Lehmer et al. 2005). ALESS57.1 and ALESS122.1 are reported as AGN candidates (Wang et al. 2013; Danielson et al. 2017), while the origin of the X-ray emission in ALESS67.1 is most probably related to SF.

ALMA observations were taken in Cycle 2 as part of the project 2013.1.00470.S (PI: Hodge), with a total integration time of $\sim 2.5 \mathrm{hr}$ in ALMA Band 3, covering the spectral range expected for the line emission of the $\mathrm{CO}(3-2)$ transition at these redshifts. The sources were observed on 2015 September 4,6 , and 20 , using the $12 \mathrm{~m}$ array and under good phase stability/weather conditions, with PWV at zenith ranging between 1.56 and $3.03 \mathrm{~mm}$. The antenna configuration consisted of 33, 36, and 35 antennas, respectively, achieving synthesized beam sizes that range between 0 " 34 and 0".67 major axis FWHM with the largest angular scales (LAS) between 1 ."9 and 2".9. The observations were calibrated based on Jupiter as the flux-calibrator, J0334-4008 as the band-pass calibrator, and J0334-401 as the phase calibrator.

New ALMA observations of ALESS49.1 (Wardlow et al. 2018) were taken during Cycle 4 on 2016 November 12, 16, and 20 as part of the project 2016.1.00754.S (PI: Wardlow). These observations were carried out using a total integration time of $\sim 2700 \mathrm{~s}$ and using the longest baselines of $\sim 650 \mathrm{~m}$. With an angular resolution of 1"! 1 , the additional data increase the signal-to-noise ratio $(\mathrm{S} / \mathrm{N})$ of our high-resolution data. We concatenated both Cycle 2 and Cycle 4 data sets achieving a combination of high-resolution imaging, high $\mathrm{S} / \mathrm{N}$ and at the same time reducing concerns whether we might have resolved any significant flux from ALESS49.1, due to the short baselines covered. An analysis of the environment of ALESS49.1 is presented in Wardlow et al. (2018).

The ALMA data were calibrated following the ALMA pipeline and using the Common Astronomy Software Application package (CASA, McMullin et al. 2007). Manual flagging of a few corrupted time windows for ALESS57.1 was required after an inspection of the calibration output. The imaging process was carried out using CASA tasks (version 4.7.0). The UV data were Fourier transformed and deconvolved from the point-spread function using the CASA CLEAN algorithm. After resampling of the visibilities at different spectral resolutions to optimize the $\mathrm{S} / \mathrm{N}$, we produced the final data cubes averaging the visibilities to channel widths of $16,61,82$, and $77 \mathrm{MHz}$ for ALESS49.1, 57.1, 67.1, 122.1, respectively. At the native resolution, the rms values achieved for the final data cubes range from 0.11 to $0.18 \mathrm{mJy}^{\text {beam }}{ }^{-1}$ (see Table 1).

Due to the high spatial resolution of the data, it is not trivial to estimate the masks on which to apply the CLEAN task. We adopted an iterative cleaning technique (e.g., Chen et al. 2017), in order to optimize the mask size estimation to include possible extended low surface brightness emission. Iterative cleaning consists of drawing concentric circular mask regions at increasing radii and applying the CLEAN task and line flux extraction within them. Plotting the resulting line fluxes against the corresponding circular region radii produces a curve of growth (see upper right panels of Figure 1). The expected behavior of the measured flux density in a curve of growth is to continuously increase as a function of radius, reaching a point of convergence at the maximum extent of the source. We used the masks inferred from the iterative cleaning method to extract the final line cubes. We explored the data for emission at different spatial scales and surface brightness, first at the native resolution using natural weighting, then using a Briggs weighting with a robustness parameter 0.5 , to image the $\mathrm{CO}$ emission at lower $\mathrm{S} / \mathrm{N}$ but slightly higher spatial resolution and finally, by tapering the visibilities to lower resolutions $(1.5-2 \times$ native beam size) to recover extended emission.

\section{2. $\mathrm{CO}(3-2)$ Line Detections and Continuum}

We detect significant $\mathrm{CO}(3-2)$ line emission for the four sources in our sample even from the dirty data cubes, i.e., in the Fourier transformed visibilities prior to the beam deconvolution (cleaning process). The $\mathrm{CO}$ detections are coincident with the expected ALMA Cycle 0 positions, and at frequencies which confirm the previously reported spectroscopic redshifts (Table 1). We identify strong line detections for all the sources in the final line cubes constructed by applying the circular cleaning masks described in Section 2.1. Figure 1 shows the data as well as Gaussian profile fits to the extracted spectra. The estimated $\mathrm{CO}(3-2)$ line parameters are summarized in Table 2. We next briefly describe some important features of the line emission of each source.

The CO spectrum of ALESS49.1 is clearly best described by a double-peaked profile and was thus fitted using an asymmetrical two-Gaussian model. The red and blue component line widths are $280 \pm 40 \mathrm{~km} \mathrm{~s}^{-1}$ and $390 \pm 30 \mathrm{~km} \mathrm{~s}^{-1}$ (FWHM), respectively, and are separated by $300 \pm 20 \mathrm{~km} \mathrm{~s}^{-1}$.

The CO spectrum of ALESS57.1 is marginally spectroscopically resolved. Nevertheless, the line width is 
Table 1

Description of the ALMA Cycle 2 Band 3 Observations and Native Beam Properties

\begin{tabular}{|c|c|c|c|c|}
\hline & ALESS49.1 & ALESS57.1 & ALESS67.1 & ALESS122.1 \\
\hline R.A. (J2000) & $03: 31: 24.72$ & $03: 31: 51.92$ & $03: 32: 43.20$ & $03: 31: 39.54$ \\
\hline Decl. (J2000) & $-27: 50: 47.1$ & $-27: 53: 27.1$ & $-27: 55: 14.3$ & $-27: 41: 19.7$ \\
\hline$z$ (opt) & 2.95 & 2.94 & 2.12 & 2.02 \\
\hline$z(\mathrm{CO}(3-2))$ & $2.943 \pm 0.001$ & $2.943 \pm 0.002$ & $2.121 \pm 0.004$ & $2.024 \pm 0.001$ \\
\hline \multicolumn{5}{|c|}{ Natural weighted imaging } \\
\hline Cleaning mask radius $\left({ }^{\prime \prime}\right)^{\mathrm{a}}$ & 1.8 & 0.8 & 2.0 & 1.0 \\
\hline Synthesized beam FWHM $\left({ }^{\prime \prime}\right)$ & $0.69 \times 0.63$ & $0.67 \times 0.60$ & $0.56 \times 0.48$ & $0.45 \times 0.35$ \\
\hline Continuum rms $\left(\mu \mathrm{Jy}\right.$ beam $\left.^{-1}\right)$ & 17.6 & 19.5 & 18.2 & 16.3 \\
\hline Channel widths in final cubes $(\mathrm{MHz})$ & $15.6^{\mathrm{b}}$ & 60.5 & 81.5 & 77.3 \\
\hline Channel widths in final cubes $\left(\mathrm{km} \mathrm{s}^{-1}\right)$ & $54^{\mathrm{b}}$ & 200 & 213 & 196 \\
\hline Channel rms $\left(\mu \mathrm{Jy}\right.$ beam $\left.^{-1}\right)$ & $182^{\mathrm{b}}$ & 148 & 113 & 131 \\
\hline
\end{tabular}

Notes.

${ }^{a}$ Chosen as explained in Figure 1.

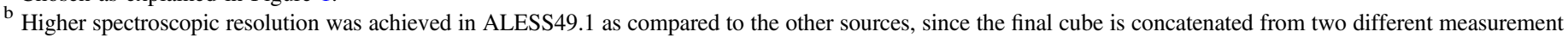
sets. See Section 2.1 for details.

appropriately approximated by a Gaussian fit and the resulting values are summarized in Table 2 .

The CO line of ALESS67.1 shows a very large line width (FWHM $\sim 800 \mathrm{~km} \mathrm{~s}^{-1}$ ) and appears asymmetric although the asymmetry is not statistically significant, due to the low $\mathrm{S} / \mathrm{N}$ in this source. We postulate that the CO line of ALESS67.1 is produced by two sources of emission. This is supported by the velocity averaged image (Figure 2), where two spatial components are recognizable independently in both $\mathrm{CO}$ (ALMA) and optical/near-infrared (HST) imaging: one main right component of around 1 arsec radius and a second extended emission in the east. We thus give preference to the description of this $\mathrm{CO}$ line as originating in two sources (possibly an early stage of a merger event) and focus on the emission extracted from the main component (1 arsec radius), which is shown as a black line overlaid on the total line emission in Figure 1.

The CO spectrum of ALESS122.1 is the brightest in our sample, and its line profile is well described by a single Gaussian fit. Table 2 lists the $\mathrm{CO}(3-2)$ line properties and bestfit parameter values. A detailed description of the velocity field sampled by these CO lines will be provided in Section 3.2.1.

Continuum images were made for all the sources after excluding the channels contributing to the $\mathrm{CO}(3-2)$ line emission. Using natural weighting to achieve the highest sensitivity, ${ }^{17}$ we obtained images with $\sigma_{\text {rms }} \sim 6,20,18$, $12 \mu \mathrm{Jy}_{\text {beam }^{-1}}$, as summarized in Table 1 . The continuum emission is detected in only two of the four sources in our data set: ALESS49.1 and ALESS122.1. In both cases, the naturally weighted dust continuum emission at $3 \mathrm{~mm}$ (rest-frame $\sim 850 \mu \mathrm{m})$ is detected at 4- $\sigma$ significance at sub-arsecond resolution (Table 2).

To test for consistency with the Cycle 0 continuum observations at $870 \mu \mathrm{m}$ (Hodge et al. 2013b), we use an extrapolation following $S_{\nu} \propto \nu^{2+\beta}$, where $S_{\nu}$ is the measured flux density and $\beta$ is the dust emissivity spectral index. Adopting $\beta=1.5$ as a reasonable assumption for dusty galaxies (Casey et al. 2014; Swinbank et al. 2014), the values

\footnotetext{
17 The rms for ALESS49.1 is achieved using the concatenated data set which has a lower resolution $\left(\theta_{\text {beam }} \sim 0^{\prime \prime}\right.$. 7 ) than that of Cycle 2 only $(\sim 0$.! 6$)$, as discussed in Section 2. The $88 \mathrm{GHz}$ continuum emission measured from the Cycle 4 low-resolution data alone $\left(\theta_{\text {beam }} \sim 1\right.$ !' 5 ) has a $\sim 9 \sigma$ detection.
}

extrapolated from $870 \mu \mathrm{m}$ are consistent with our measured $S_{3 \mathrm{~mm}}$ peak flux densities and upper limits, which are listed in Table 2 .

\subsection{Source Size Estimation}

High-resolution imaging reveals the detailed structure of the gas and dust continuum emission, which is key for placing constraints on the dynamical states of the sources. However, high-resolution interferometry is less sensitive to extended, low surface brightness emission, and thus care must be exercised in the estimation of the source sizes (e.g., Emonts et al. 2016; Dannerbauer et al. 2017; Ginolfi et al. 2017). Although the iterative cleaning method presented in Section 2.1 can provide a sense of the total source extent, it is prone to correlated noise effects and consequently may yield uncertain results. Moreover, iterative cleaning can only provide an (intrinsic) source size estimate when the extent of the source is greater than the synthesized beam. To determine the sizes independently of these possible beam-convolution effects intrinsic to the imaging process, we estimate the sizes directly from the UV data (lower-right panels of Figure 1).

We extract the UV data corresponding to the frequencies within the FWHM of the line from the continuum-subtracted cube, centered at the $\mathrm{CO}(3-2)$ observed frequency. For each source, we then average the visibilities at different UV distances and plot the resulting amplitudes against them, as shown in Figure 1, where the data points correspond to the mean amplitude of the visibilities within each bin and the errors are the corresponding standard deviation of the mean. We fit a Gaussian profile to the data, taking into account the uncertainties, and measure the half-light radius of the source, which corresponds to the radius within which half of the light of the galaxy is contained. We report the estimated half-light radii $r_{1 / 2}$ in Table 2 and adopt these values for our analysis. The half-light radii of our sources range between 0 " 4 and 0 ". 8 , which correspond to $2.6-6.9 \mathrm{kpc}$ at the respective redshifts, implying total physical sizes (FWHM) of $\sim 10 \mathrm{kpc}$. In a study of the $\mathrm{CO}(3-2)$ emission of a similar sample of SMGs at $z \sim 2-3$, Tacconi et al. (2008) found sizes ranging between 2 and $12 \mathrm{kpc}$. More recent high-resolution molecular gas studies have reported similar sizes for low- $J(J \leqslant 3) \mathrm{CO}$ emission 

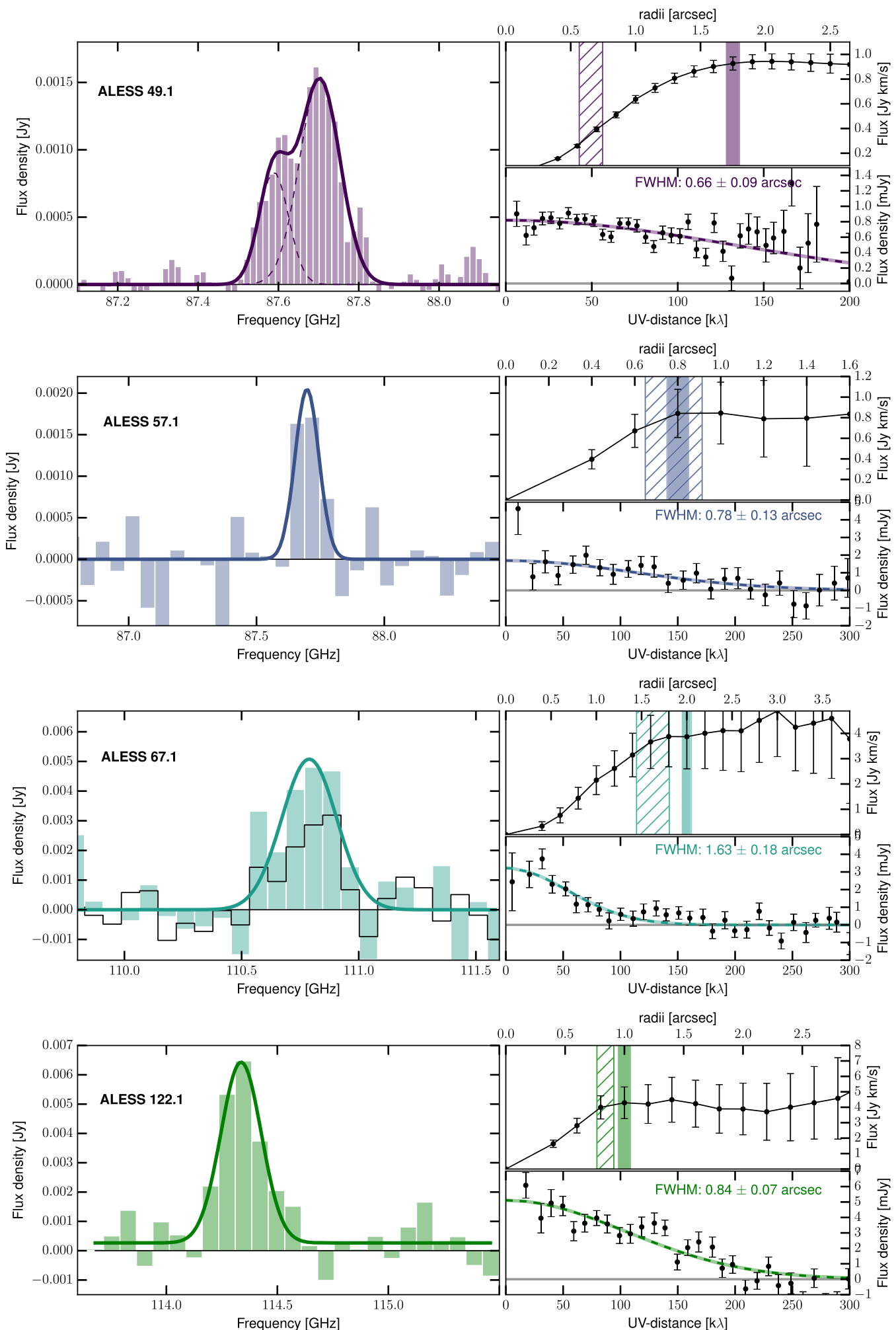

Figure 1. Analysis of the $\mathrm{CO}(3-2)$ data: The left panels show the $\mathrm{CO}(3-2)$ spectra of our sources. The spectral resolution is $\sim 54,200,213$, and $116 \mathrm{~km} \mathrm{~s} \mathrm{~s}^{-1}$, for ALESS49.1, ALESS57.1, ALESS67.1, and ALESS122.1, respectively. Gaussian fits to the spectra are shown by the solid lines. For ALESS49.1 we fit a twocomponent Gaussian profile and show each component as dotted lines. For ALESS67.1, we additionally show the CO spectrum extracted exclusively from within a mask of $\sim 1^{\prime \prime}$ around the centroid of the main component as a black line (see Section 2.2 for more details). The right panels show two methods used to calculate the cleaning masks and intrinsic source sizes, respectively. The first method (top right) is a curve of growth analysis, where the shaded area shows the radius at convergence. This method was used to determine the size of the area used for masking the cleaning process. The second method (bottom right) is the analysis of the visibilities (UV) profiles, which reliably estimate intrinsic source sizes. A single Gaussian fit to the UV data is shown by the line. The half-light radius $r_{1 / 2}$ from the UV fitting method is a more robust estimate of the true size of the sources, since the curve of growth analysis is prone to be affected by correlated noise. We draw hashed areas to show the FWHM from the Gaussian fit (i.e., $2 \times r_{1 / 2}$ ) on the top-right panel and see that in most cases, it roughly corresponds to the peak of the curve of growth. 
Table 2

CO(3-2) Line Properties and Estimated Quantities

\begin{tabular}{|c|c|c|c|c|}
\hline & ALESS49.1 & ALESS57.1 & ALESS67.1 & ALESS122.1 \\
\hline $\mathrm{CO}(3-2) r_{1 / 2}(\mathrm{kpc})$ & $2.6 \pm 0.4$ & $3.1 \pm 0.5$ & $6.9 \pm 0.8$ & $3.6 \pm 0.3$ \\
\hline $\mathrm{FWHM}_{\mathrm{CO}(3-2)}\left(\mathrm{km} \mathrm{s}^{-1}\right)$ & $610 \pm 30$ & $360 \pm 90$ & $720 \pm 160(500 \pm 110)^{\mathrm{a}}$ & $600 \pm 80$ \\
\hline Dust continuum $S_{3 \mathrm{~mm}}(\mu \mathrm{Jy})$ & $34 \pm 6$ & $54 \pm 18$ & $\leqslant 54^{\mathrm{b}}$ & $\leqslant 60^{\mathrm{b}}$ \\
\hline Inclination $i$ (degrees $)^{\mathrm{c}}$ & $80 \pm 30$ & $40 \pm 50$ & $50 \pm 20$ & $40 \pm 20$ \\
\hline Velocity integrated flux density $I_{\mathrm{CO}(3-2)}\left(\mathrm{Jy} \mathrm{km} \mathrm{s}^{-1}\right)$ & $0.89 \pm 0.07$ & $0.8 \pm 0.2$ & $3.9 \pm 1.2(1.8 \pm 0.5)^{\mathrm{a}}$ & $4.2 \pm 0.8$ \\
\hline Velocity integrated flux density $I_{\mathrm{CO}(1-0)}\left(\mathrm{Jy} \mathrm{km} \mathrm{s}^{-1}\right)$ & $\ldots$ & $\cdots$ & $0.44 \pm 0.08^{\mathrm{d}}$ & $0.64 \pm 0.07^{\mathrm{d}}$ \\
\hline $\mathrm{CO}(3-2)$ line luminosity $L_{\mathrm{CO}(3-2)}^{\prime}\left(10^{11} \mathrm{~K} \mathrm{~km} \mathrm{~s}^{-1} \mathrm{pc}^{2}\right)$ & $0.39 \pm 0.03$ & $0.4 \pm 0.1$ & $1.0 \pm 0.3(0.5 \pm 0.1)^{\mathrm{a}}$ & $1.0 \pm 0.2$ \\
\hline $\mathrm{CO}(1-0)$ line luminosity $L_{\mathrm{CO}(1-0)}^{\prime}\left(10^{11} \mathrm{~K} \mathrm{~km} \mathrm{~s}^{-1} \mathrm{pc}^{2}\right)$ & $0.5 \pm 0.2^{\mathrm{e}}$ & $0.5 \pm 0.2^{\mathrm{e}}$ & $1.0 \pm 0.2^{\mathrm{d}}$ & $1.3 \pm 0.2^{\mathrm{d}}$ \\
\hline Molecular gas mass $\left(10^{11} M_{\odot}\left(\alpha_{\mathrm{CO}}=1.\right)\right)$ & $0.5 \pm 0.2$ & $0.5 \pm 0.2$ & $1.0 \pm 0.2$ & $1.3 \pm 0.2$ \\
\hline$M_{\mathrm{dyn}}\left(r \leqslant 2 r_{1 / 2}\right)\left(10^{11} M_{\odot}\right)$ & $1.1 \pm 0.2$ & $1.1 \pm 0.5$ & $3.6 \pm 1.6$ & $5.3 \pm 1.6$ \\
\hline Stellar mass ${ }^{\mathrm{f}}\left(10^{11} M_{\odot}\right)$ & $0.4 \pm 0.1$ & $0.8 \pm 0.1$ & $2.4 \pm 2.1$ & $0.8 \pm 0.5$ \\
\hline Infrared luminosities $L_{\mathrm{IR}(3-2000 \mu \mathrm{m})}\left(\times 10^{12} L_{\odot}\right)^{\mathrm{f}}$ & $6.8 \pm 0.6$ & $2.3 \pm 2.2$ & $5.0 \pm 1.5$ & $8.3 \pm 2.5$ \\
\hline $\operatorname{SFR}^{\mathrm{f}}\left(M_{\odot} \mathrm{yr}^{-1}\right)$ & $700 \pm 100$ & $200 \pm 200$ & $400 \pm 100$ & $700 \pm 200$ \\
\hline
\end{tabular}

Notes.

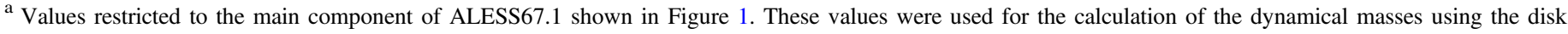
approximation in Section 3.3.

b $3 \sigma$ upper limits.

${ }^{c}$ Computed from axial ratios estimated with the CASA task IMFIT.

d Measured by Huynh et al. (2017).

e Estimated using $r_{3 / 1}=0.78 \pm 0.27$ (Sharon et al. 2016).

${ }^{\mathrm{f}}$ As presented by da Cunha et al. (2015).

(e.g., Ivison et al. 2011; Riechers et al. 2011; Hodge et al. 2012). It is interesting to note that the available high- $J$ CO observations for some of these sources $(J=6$; Engel et al. 2010) reveal that the physical extent of the emission may decrease for higher $J$-transitions.

Figure 2 shows the $\mathrm{CO}(3-2)$ emission overlaid on the stellar emission as traced by the WFC3/IR (bands $H_{160}, J_{125}, Y_{105}$ ) and/or ACS imaging $\left(I_{814}\right)$ from HST (e.g., Chen et al. 2015). We note that the astrometry in the HST images has been corrected based on the GAIA star positions, which are aligned to the Fifth Fundamental Catalog (FK5) (Gaia Collaboration et al. 2016). With the exception of ALESS122.1, the CO gas overlaps with the stellar distributions, although they have slight offsets (0." $1-0$. . $3,1-3 \mathrm{kpc}$ offsets). The sizes of the gas and stellar distribution in these sources are also roughly similar. Specifically, the ratio of the $H S T H_{160}$ imaging (Chen et al. 2015) to CO half-light radii of these ALESS sources ranges between 1 and 1.5 (ALESS122.1 not included here as no $H_{160}$ is available). We will compare the extent of the different components in a statistical study for SMG populations in Section 4.2. ALESS122.1 displays the striking feature that the gas and stellar emission are completely offset. We must point out, however, that the stellar emission in ALESS122.1 is exclusively represented by the ACS $I_{814}$ band imaging (restframe $270 \mathrm{~nm}$ ), in contrast to the other three sources which are covered by at least one WFC3/IR band. This particular case will be discussed in detail in Section 4.1.

Previous studies have shown that submillimeter continuum observations of similar galaxy populations reveal very compact rest-frame far-infrared-emitting regions, with median half-light radii of only $\sim 0.7-1.6 \mathrm{kpc}$ (e.g., Ikarashi et al. 2015; Simpson et al. 2015; Hodge et al. 2016; Oteo et al. 2017). This is significantly more compact than the molecular gas emission in our sources. This difference in compactness is similarly observed between the dust continuum and radio emission of SMGs. Median radii of radio emitting regions originating from SMGs have been reported to be around $2.1 \mathrm{kpc}$ on average (e.g., Chapman et al. 2004; Biggs \& Ivison 2008; Miettinen et al. 2015, 2017). Motivated by these differences, we will explore in detail the relation between the radial profiles of the molecular gas, the dust continuum emission (at rest-frame $250 \mu \mathrm{m}$ ) and stellar emission in Section 4.2.

\section{Dynamical Constraints to the Total Gas Masses}

\subsection{Molecular Gas Masses}

The masses of the $\mathrm{CO}(3-2)$ emitting gas in our sources can be estimated from the observed ${ }^{12} \mathrm{CO}(J=3-2)$ line luminosities $L_{\mathrm{CO}}^{\prime}$. These were calculated following Solomon \& Vanden Bout (2005) as $L_{\mathrm{CO}}^{\prime}=3.25 \times 10^{7} S_{\mathrm{CO}} \Delta v \nu_{\mathrm{obs}}^{-2} D_{\mathrm{L}}^{2}(1+z)^{-3} \mathrm{~K} \mathrm{~km} \mathrm{~s}^{-1} \mathrm{pc}^{2}$, where $S_{\mathrm{CO}} \Delta v$ is the velocity integrated flux, in $\mathrm{Jy} \mathrm{km} \mathrm{s}^{-1} ; \nu_{\mathrm{obs}}$ is the observed frequency, in $\mathrm{GHz}$; and $D_{\mathrm{L}}$ is the luminosity distance in Mpc. The resulting values are listed in Table 2. Through an extrapolation from these $\mathrm{CO}$ masses we can calculate the total molecular content of the systems (dominated by $\mathrm{H}_{2}$ ) by assuming a $\mathrm{CO}$ line luminosity to $\mathrm{H}_{2}(+\mathrm{He})$ mass conversion factor, $\alpha_{\mathrm{CO}}$, and a brightness temperature ratio of $\mathrm{CO}(J=3-2)$ to $\mathrm{CO}(J=1-0)$. However, the combination of spatial and spectroscopic resolution of our data allows us to make an estimate of the total gas mass independently of the abovementioned assumptions, by estimating kinematic parameters (Section 3.2) and using further multiwavelength information to estimate the stellar mass contribution (Section 3.4). But to begin with we use the classical approach to estimate the molecular gas masses as a function of $\alpha_{\mathrm{CO}}$.

Using the measurements of ${ }^{12} \mathrm{CO}(J=1-0)$ by Huynh et al. (2017) for ALESS67.1 and ALESS122.1 (see Table 2), we calculate their brightness temperature ratio $r_{3 / 1}=L_{\mathrm{CO}(3-2)} /$ $L_{\mathrm{CO}(1-0)}$, which yields $r_{3 / 1}=1.01 \pm 0.36$ and $r_{3 / 1}=0.77 \pm$ 0.19 , respectively. This is consistent with previous estimates for the SMG population (Ivison et al. 2011; Bothwell et al. 2013; Sharon et al. 2016). For ALESS49.1 and ALESS57.1 we estimate the ${ }^{12} \mathrm{CO}(1-0)$ emission using the excitation ratio for SMGs derived by Sharon et al. (2016), $r_{3 / 1}=0.78 \pm 0.27$, 

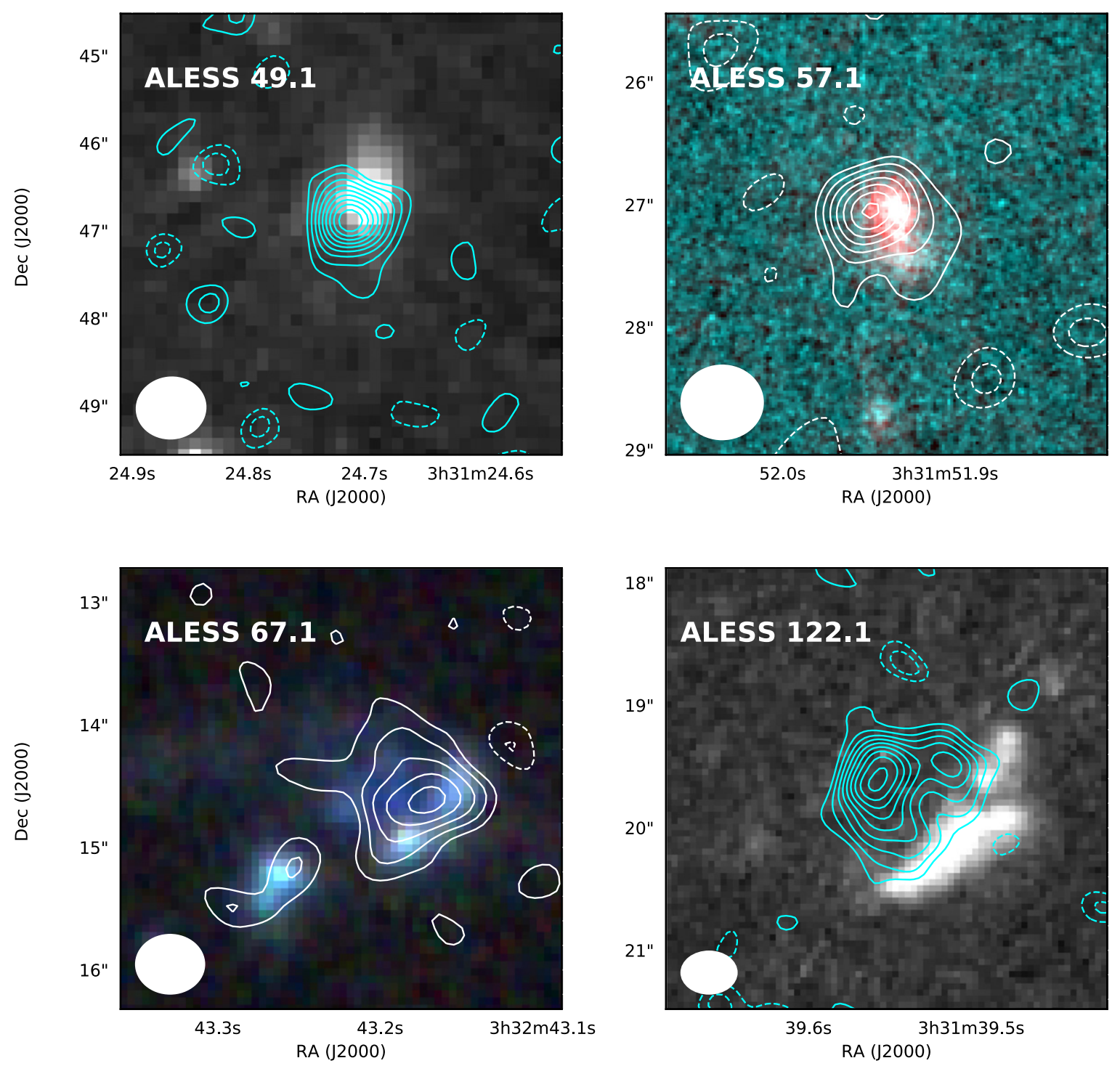

Figure 2. $\mathrm{CO}(3-2)$ velocity averaged contours overlaid on optical/near-IR cutouts from the available $H S T$-WFC 3 and/or ACS imaging. Resolutions of 0 ". $4-0$ ". 6 are achieved through natural weighting. The contours are obtained by averaging over the velocity range corresponding to the FWHM of the Gaussian fit to the CO spectrum. The contours in all of the maps show $2-10 \sigma_{\mathrm{rms}}$ regions, where the $\sigma_{\mathrm{rms}}$ for the velocity averaged images is: $\left(0.10 \mathrm{mJy}^{-1}\right.$ beam $\left.{ }^{-1}\right)$ for ALESS49.1, $(0.15 \mathrm{mJy}$ beam $\left.^{-1}\right)$ for ALESS57.1, $\left(0.11 \mathrm{mJy}_{\text {beam }}{ }^{-1}\right)$ for ALESS67.1, and $\left(0.13 \mathrm{mJy}_{\text {beam }}^{-1}\right)$ for ALESS122.1. The beam sizes are shown in the lower-left corners of the images. The background map for ALESS49.1 represents the $H_{160}$ imaging, the two-color map for ALESS57.1 uses the $H_{160}$ and $I_{814}$ fluxes, the background r-g-b map for ALESS67.1 represents $H_{160}, J_{125}, Y_{105}$ and the background map for ALESS122.1 shows the $I_{814}$ imaging. The $H S T$ imaging has been corrected for astrometric offsets using GAIA data. We see that our observations strongly detect and resolve the $\mathrm{CO}$ emission of these sources on scales of 3-5 kpc, finding different morphologies in both $\mathrm{CO}$ and stellar emission.

yielding $L_{\mathrm{CO}(1-0)}^{\prime}=(0.5 \pm 0.2) \times 10^{11} \mathrm{~K} \mathrm{~km} \mathrm{~s}^{-1} \mathrm{pc}^{2}$, for both sources. The derived molecular gas masses as a function of $\alpha_{\mathrm{CO}}$ (equivalent to $\alpha_{\mathrm{CO}}=1$ ) are reported in Table 2 .

In order to investigate the possible effects of the presence of an AGN on the molecular gas in our sample, we explore possible correlations between galaxy properties inferred from the CO measurements (listed in Table 2). We test for any bimodality in the distribution of properties such as the FWHM of the CO line, the gas-to-stellar mass fraction and SF efficiency $\left(\mathrm{SFE}=\mathrm{SFR} / M_{\mathrm{gas}}\right.$ ) corresponding to sources with and without an AGN (ALESS57.1 and ALESS122.1 are the AGN-host candidates in our sample, see Section 2.1). We find no clear bimodality correlated to the presence or absence of an AGN, although we note that this result is not conclusive given the very small size of our sample. However, we expect that the scales probed by our $\mathrm{CO}$ observations are not affected by
AGN, since the CO transition probed $(J=3-2)$ is not expected to be a tracer of AGN excitation (e.g., Sharon et al. 2016).

\subsection{CO Line Kinematics}

The morphological and kinematic properties of our resolved $\mathrm{CO}$ images allow us to explore the nature of these star-forming systems, whether these are in an early stage of a merger with a chaotic structure, or if their velocity fields are described by an ordered rotating disk. It is important to note, however, that these scenarios are not mutually exclusive. Observing dynamics consistent with a rotating disk does not preclude the galaxy from being a merging system, as the gas is collisional and an ordered rotating disk can quickly reform after the final coalescence stage as has been shown observationally 

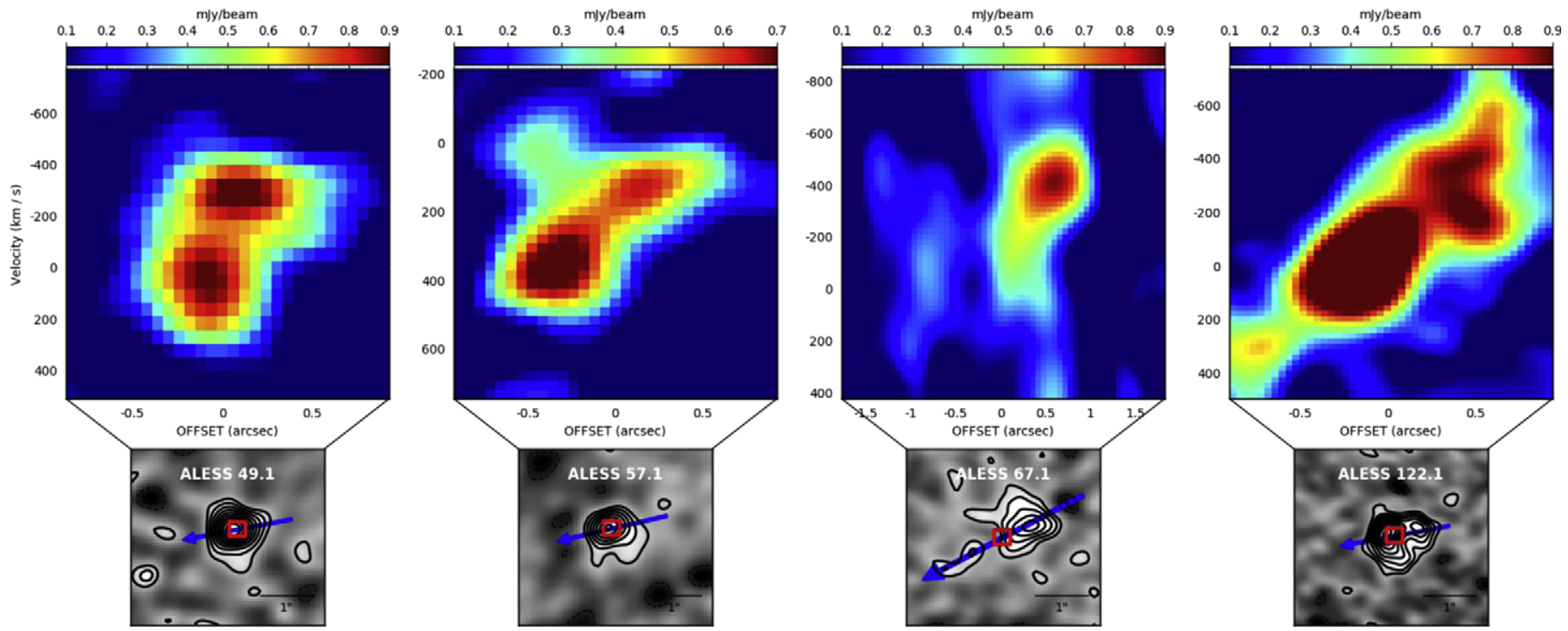

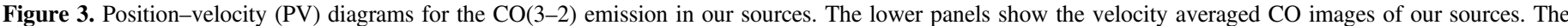

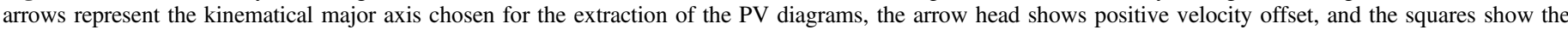

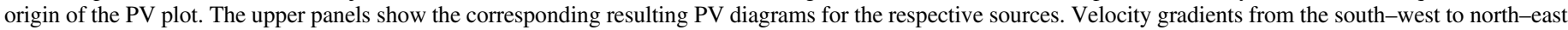

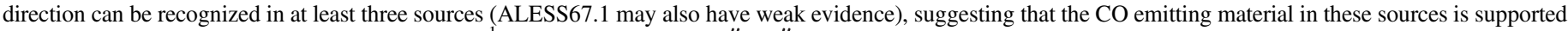
by rotation with velocity gradients of $\sim 300 \mathrm{~km} \mathrm{~s}^{-1}$ on spatial scales of $\sim 0$. $2-0$.. 7 .

and theoretically (Robertson et al. 2006; Hopkins et al. 2009, 2013; Ueda et al. 2014).

While the few existing examples of $\mathrm{CO}$ detections at a similar resolution suggest a mixture of mergers and disk-like motion (e.g., Tacconi et al. 2008; Engel et al. 2010; Hodge et al. 2012), recent high-resolution continuum imaging of SMGs has shown that the dust continuum emission (at restframe $250 \mu \mathrm{m}$ ) is mostly described by compact disk profiles (e.g., Simpson et al. 2015; Hodge et al. 2016). Here we use our high-resolution $\mathrm{CO}$ observations to directly test the kinematics of these systems.

\subsubsection{Position-Velocity (PV) Diagrams}

To estimate the kinematic properties of our sources we first investigate their PV diagrams. We apply the interactive PV Diagram Creation task from the CASA VIEWER to the data cubes of our sources with spectral resolution corresponding to channel widths of $\sim 100 \mathrm{~km} \mathrm{~s}^{-1}$. The lower panel of Figure 3 shows the kinematical major axis chosen for the extraction of the PV diagrams overplotted as blue arrows on the velocity averaged $\mathrm{CO}$ images. The upper panels show the resulting PV diagrams for the respective sources. We find velocity gradients in the PV diagrams of three of our four sources (with ALESS67.1 also showing some weak signs), suggesting that the bulk of emission in these sources is dominated by rotation.

The velocity gradients of ALESS49.1, ALESS57.1 show double peaks at each side of the galactic center, although the detailed velocity structure is ambiguous given the modest $\mathrm{S} / \mathrm{N}$. In ALESS49.1, the double-peaked spatial structure gives rise to the double-peaked line profile observed in Figure 1. Since both sources have the CO centered on a single optical nucleus (as seen from their high-resolution HST imaging), the velocity structure suggests the presence of a disk structure or a disk-shaped merger remnant, rather than an early stage merger.

The CO emission in ALESS67.1 and its velocity structure appears more chaotic, which is probably accentuated by the combination of extended emission and the lower $\mathrm{S} / \mathrm{N}$ achieved in this source. A detailed analysis of the velocity structure of this individual source has been conducted in a complementary study by Chen et al. (2017). They presented SINFONI $\mathrm{H \alpha}$ observations of ALESS67.1, revealing that the extended $\mathrm{CO}$ emission (including the second component to the south-east) follows the bulk rotational motion of the rest-frame optical $\mathrm{H} \alpha$ emission line. Kinematic modeling of both lines concluded the bulk of the molecular gas in ALESS67.1 could be described as an ongoing merger, although without being able to reject a rotating disk given the errors. It is still uncertain whether ALESS67.1 is a multicomponent system in an early state of merging. This scenario has been discussed in Section 2.2 based on our $\mathrm{CO}$ and HST observations, and it has also been supported by the analysis of its kinematics based on ancillary data by Chen et al. (2017). For further dynamical mass calculations we will only consider the properties of the most luminous component of ALESS67.1 (Table 2) and assume that this component is well described by a rotating disk.

ALESS122.1 is the source with highest $\mathrm{S} / \mathrm{N}$ in our sample and its velocity structure is consistent with disk rotation. Due to these reasons it was possible to analyze its velocity field quantitatively using a rotating disk model, as will be described in Section 3.2.2.

Although the velocity gradients may be consistent with large-scale disk rotation as a bulk motion, we emphasize that no conclusions can be drawn to exclude complex, disturbed gas motions on scales smaller than the resolution limit of our data. Based on this qualitative analysis of the PV diagrams in Figure 3, we will adopt the scenario of a rotating disk for our sources for the computation of their dynamical masses. The velocity line width (and thus an estimate of the velocity dispersion) and other morphological properties will be adopted from the values listed in Table 2 .

\subsubsection{Kinematic Modeling}

To quantitatively describe the kinematics of the molecular gas in our sources, we model the data using the modeling 


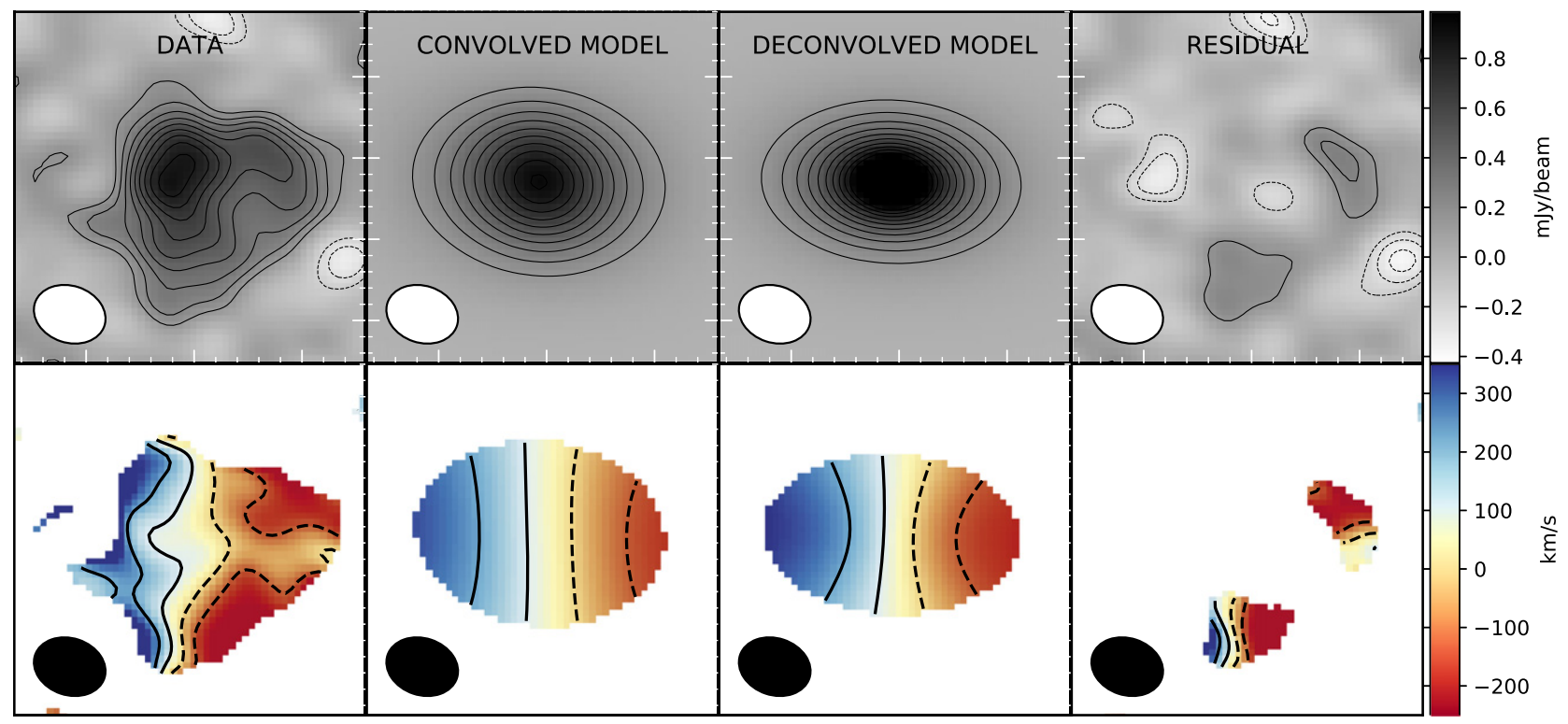

Figure 4. Modeling of the morphology and kinematics of ALESS122.1 with GALPAK3D. The top row shows the zeroth-moment maps (integrated intensity) of the observed, convolved model, deconvolved model and residual cubes. The contour lines in the top row show the $2-9 \sigma_{\text {rms }}$ levels, where $\sigma_{\text {rms }}$ is the noise level of the zeroth-moment maps. The bottom row shows the first-moment maps (intensity-weighted velocity) of the corresponding cubes, showing only regions with fluxes $>2 \sigma_{\text {rms. }}$. The color scale represents the width of the $\mathrm{CO}$ line in $\mathrm{km} \mathrm{s}^{-1}$. The low-intensity levels of the residual maps show that the bulk of the emission can be described by the disk rotation. This, however, does not preclude the galaxy from being a merger, as an ordered rotating disk can be quickly "reformed" after the final coalescence stage in a few dynamical times (Robertson et al. 2006; Hopkins et al. 2013).

package GALPAK3D. GALPAK3D is a Bayesian parametric Markov Chain Monte Carlo fitter for three-dimensional (3D) galaxy data that attempts to disentangle the galaxy kinematics from resolution effects (Bouché et al. 2015). Starting from a set of uniform priors on the disk parameters (source center, radius, inclination, velocity dispersion, etc.,) a 3D disk galaxy model is produced assuming an exponential radial flux profile. The fitting process consists then in comparing the observed data cube to mock data cubes modeled at each point of the parameter space. In each step the reduced $\chi_{\nu}^{2}$ value is then minimized to efficiently sample the probability density functions of the parameters. The reliability of the inferred kinematic parameters in GALPAK3D goes approximately as $\frac{\delta p}{p} \propto \frac{r_{1 / 2}}{r_{\text {beam }}} \times \mathrm{SB}_{1 / 2, \text { obs }}$, i.e., the uncertainty in the inference of the parameter $p$ is inversely proportional to the source size to resolution ratio $\left(\frac{r_{1 / 2}}{r_{\text {beam }}}\right)$ and surface brightness of the source $\left(\mathrm{SB}_{1 / 2, \mathrm{obs}}\right)$. Given the dependence of these uncertainties on the quality of the data and due to the $\mathrm{S} / \mathrm{N}$ limits in most of our sources, we were able to apply this method only to the source with the highest $\mathrm{S} / \mathrm{N}$ and resolution, ALESS122.1. For the sources on which the dynamical modeling cannot be applied, we use the line profiles to draw estimates of kinematic properties such as rotational velocity (Section 3.3).

Figure 4 shows zeroth-moment (integrated intensity) and firstmoment (intensity-weighted velocity) maps for ALESS122.1. The four images correspond to the moment maps of the observed data cube, the convolved model, deconvolved model and residual (model-substracted) cubes, where the latter three cubes are output products of GALPAK3D. We estimated the noise per pixel in the zeroth-moment map as $\sqrt{N} \times \sigma_{\text {chan }}$, where $N$ is the number of channels used and $\sigma_{\text {chan }}$ is the rms noise per channel. Using this value, for the computation of the first-moment maps of the four cubes, we masked out all pixels with $\mathrm{S} / \mathrm{N}<2$. The bottom row of Figure 4 shows the first-moment maps (intensity-weighted velocity) of the corresponding cubes. The color scale has been chosen to represent the velocity width of CO line of the source.

The resulting 3D model cube shows a modest agreement with the data, with a reduced $\chi_{\nu}^{2}$ of 1.87 , which is calculated over the total area and frequency range covered by the observed and modeled data cubes, as represented in Figure 4. The slightly high, reduced $\chi_{\nu}^{2}$ value can be explained by the residual structure, which can be seen in the 0th and 1st moment maps of the residual cubes. The model parameters obtained for the source agree with comparable properties inferred directly from the line profile.

The bulk of the velocity field of ALESS122.1 is consistent with a rotation-dominated disk (mindful of the residual clumps), with an inclination of $i \sim 52^{\circ}$, a maximum rotational velocity of $v_{\max } \sim$ $560 \mathrm{~km} \mathrm{~s}^{-1}$, and a velocity dispersion of $\sigma_{v} \sim 130 \mathrm{~km} \mathrm{~s}^{-1}$. The residual image in the right upper panel of Figure 4 reveals few residual clumps between 2 and $3 \sigma$ significance, consistent with noise. We conclude that the bulk of the velocity field is to a first order consistent with disk rotation.

\subsection{Dynamical Masses}

The kinematic properties of a galaxy, obtained e.g., through modeling its velocity field, can provide a reliable estimation of the mass enclosed within the region covered by the emitting medium. At high redshift, this is a complicated task, since observations of molecular gas are frequently poorly spatially resolved and the morphology of the mass distributions are thus usually unknown. Based on the kinematic study above and the size estimates from our analysis, we calculate the dynamical masses assuming the bulk of the emission in our sources can be well described by a rotating disk. The total dynamical mass within a radius $r=2 \mathrm{r}_{1 / 2}$ is then given by:

$$
M_{\mathrm{dyn}}\left(r<2 r_{1 / 2}\right)=\frac{\left(v_{\max } / \sin (i)\right)^{2} \times 2 r_{1 / 2}}{G},
$$


where $r_{1 / 2}$ is the half-light radius estimated through UV fitting measured in kpc, $i$ is the inclination of the galaxy (2), and $G$ the gravitational constant (e.g., Solomon \& Vanden Bout 2005; Erb et al. 2006; de Blok \& Walter 2014). In cases where the velocity field cannot be modeled and $v_{\max }$ cannot be directly inferred, we can use the approximation that the velocity width is twice the maximum rotational velocity and thus we can replace $v_{\max }=1 / 2 \times \Delta V_{\mathrm{FWHM}}$ in Equation (1). This is a good assumption for a flat rotation curve with a steep inner rise and a filled exponential disk as shown by de Blok \& Walter (2014). For ALESS122.1 we use the parameter values for $v_{\max }$ resulting from the kinematic modeling in Section 3.2.2, although both estimates provide consistent results within errors.

A large uncertainty in the estimation of the dynamical masses is contributed by the inclination angle of the disk. In previous dynamical mass studies, it has been customary to adopt a value of $\left\langle\sin ^{2}(i)\right\rangle=2 / 3$ (corresponding to $i=54.7 \mathrm{deg}$ ), which is the average value expected for a randomly oriented population of disk galaxies. However, given our high-resolution data, we have enough information to use a simple assumption motivated by the early observation that the apparent axis ratio is closely related to the inclination angle for a disk. We use the relation $\cos ^{2}(i)=$ $\left((b / a)^{2}-q_{0}^{2}\right)\left(1-q_{0}^{2}\right)^{-1}$ (Hubble 1926), where $(b / a)$ is the axis ratio and $q_{0}$ is the inherent thickness of the disk, assuming $q_{0}=0.1$ (Nedyalkov 1993). Obtaining the deconvolved axis ratios using the CASA routine IMFIT, we compute the inclination angles for ALESS49.1, ALESS57.1, ALESS67.1, and ALESS122.1 to be $i \sim 80^{\circ} \pm 30^{\circ}, 40^{\circ} \pm 50^{\circ}, 50^{\circ} \pm 20^{\circ}$, and $40^{\circ} \pm 20^{\circ}$, respectively. For ALESS122.1, where a more complex analysis was possible in Section 3.2, we will adopt the inclination angle estimated through the kinematic modeling, $i \sim 52^{\circ} \pm 2^{\circ}$, which is in agreement with the axis ratio approximation within the errors. Although this is an approximation and the uncertainties are large, this estimation is superior to assuming the single value of $\left\langle\sin ^{2}(i)\right\rangle=2 / 3$ for all systems, since the inclination in individual sources may anti-correlate with the line width. It is interesting to note the high inclination angle of ALESS49.1, which is also supported by the small $\mathrm{CO}$ size and double-peaked line profile. Although these profiles are not uncommon for rotating disks and have been found in at least $40 \%$ of SMGs (Tacconi et al. 2008; Bothwell et al. 2013), the asymmetry between the double-peaked profile suggests a non-uniform distribution of the gas in the "disk," produced possibly by minor instabilities or an unresolved merger of two gas disks.

The resulting dynamical masses for our sample range from 1 to $5 \times 10^{11} M_{\odot}$ (Table 2 ), as calculated within $2 \times$ the halflight radii of the sources $r_{1 / 2}$. These values are in general agreement with previous measurements of the dynamical masses of two of these sources based on other tracers (CO $(J=1-0)$ and $\mathrm{H} \alpha$, Chen et al. 2017; Huynh et al. 2017). ${ }^{18}$ This range corresponds to masses at the high end of the average values found for other SMG samples by Ivison et al. (2010)

\footnotetext{
$\overline{18}$ In a study of $\mathrm{CO}(1-0)$ emission, Huynh et al. (2017) found equivalent dynamical masses of $M_{\text {dyn }} \sin i^{2}=(2.1 \pm 1.1)$ and $(3.2 \pm 0.9) \times 10^{11} M_{\odot}$ for ALESS122.1 and ALESS67.1, respectively. Although discrepancies were expected given that they used optical instead of $\mathrm{CO}$ extensions due to the low resolution of their $\mathrm{CO}$ data and assumed an inclination angle of $\sin ^{2}(i)=2 / 3$, their result is consistent with ours given the errors. Similarly, a dynamical study

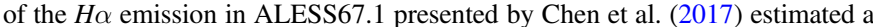
dynamical gas mass of $M_{\text {dyn }}=(2.2 \pm 0.6) \times 10^{11} M_{\odot}$ for ALESS67.1. Although this estimation resulted from the analysis of the total source, while our calculation used only the main component of the source, the values estimated agree with our calculations within the errors.
}

$\left(2.3 \times 10^{11} M_{\odot}\right)$, Tacconi et al. $(2008)\left(1.3 \times 10^{11} M_{\odot}\right)$, Engel et al. $(2010)\left(\sim 1.9 \times 10^{11} M_{\odot}\right)$ and slightly below the values found for some extreme sources such as GN20 (5.4 \pm $2.4 \times 10^{11} M_{\odot}$, Hodge et al. 2012) and SMMJ131201 (9.5 \pm $2.4 \times 10^{11} M_{\odot}$, Engel et al. 2010). The uncertainties in these values are propagated from all of the parameters used in their calculation, including the inclination angles $i$ (Table 2).

\subsection{Implications on $\alpha_{C O}$ and $\mathrm{M}_{*} / L$ Estimates}

The dynamical mass estimates discussed in Section 3.3 can be related to the various mass components in galaxies following:

$$
M_{\text {dyn }}\left(r \leqslant 2 r_{1 / 2}\right)=M_{\text {baryons }}\left(r \leqslant 2 r_{1 / 2}\right)+M_{\mathrm{DM}}\left(r \leqslant 2 r_{1 / 2}\right),
$$

where $M_{\text {baryons }}=M_{\mathrm{gas}}+M_{*}$ and $M_{\mathrm{DM}}$ is the DM contribution. Thus, given assumptions on the DM content and stellar masses, the dynamical mass estimates can be used as an independent method to constrain the total gas masses in galaxies. Before we proceed to constrain the gas masses using this method, we summarize the unknown parameters intrinsic to this calculation. We note that given the scarcity of $\mathrm{CO}(J \leqslant 3)$ gas data at high resolution, most of the existing literature on molecular gas in high-redshift galaxies usually assume fixed values for unknown parameters, estimating gas masses which are thus sensitive to these assumptions and lacking information on the inherent systematic uncertainties.

To begin with, the $\mathrm{H}_{2}$ molecules that comprise the bulk of the molecular gas reservoir in galaxies are not directly observable. Measurements of molecular gas thus rely on $\mathrm{CO}$ observations, via a conversion from the ground state $\mathrm{CO}(1-0)$ luminosity which is parametrized with the factor $\alpha_{\mathrm{CO}}$. Indeed, high-redshift measurements are providing increasing evidence that the $\alpha_{\mathrm{CO}}$ values in the early universe can be lower than in solar-metallicity galactic disks $\left(\alpha_{\mathrm{CO}} \sim 0.8-1.0\right.$,e.g., Tacconi et al. 2008; Hodge et al. 2012; Bothwell et al. 2013).

In addition to the uncertainties in the total gas mass estimation, stellar mass estimates are typically obtained via SED fitting, which relies on assumptions regarding the star formation history (SFH) of the stellar populations and dust attenuation, and can be uncertain especially for starburst systems (such as SMGs). In particular, it has been increasingly observed that SED fitting of starburst systems suffers from a degeneracy between the SFH, the mass-to-light ratio $\left(M_{*} / L_{H}\right)$ and the age of the galaxy (Hainline et al. 2011; Michałowski et al. 2012; Simpson et al. 2014). This degeneracy means that in a galaxy with an instantaneous burst SFH the total stellar mass would be contained almost exclusively within young luminous stellar populations, resulting in a low $\left(M_{*} / L_{H}\right) \sim$ 0.05. Conversely, in a galaxy of intermediate age with a constant SFH, most of the stellar mass would be distributed in old faint populations whose emission is outshone by the luminous newly born stars, implying a higher mass-to-light ratio $\left(M_{*} / L_{H} \sim 0.3\right)$. As shown by recent resolved SED fitting studies (Sorba \& Sawicki 2018), this effect could underestimate total stellar masses by factors of up to 5, especially in sources of high specific SF rates such as SMGs.

Rather than adopting a single $\left(M_{*} / L_{H}\right)$ or $\alpha_{\mathrm{CO}}$, we can instead parametrize the baryonic mass as:

$$
M_{\text {baryons }}=L_{H} \times M_{*} / L_{H}+\alpha_{\mathrm{CO}} \times L_{\mathrm{CO}},
$$


where $L_{H}$ is the rest-frame $H$-band luminosity corrected for dust obscuration (here estimated by da Cunha et al. 2015) and $L_{\mathrm{CO}}$ is the $\operatorname{CO}(J=1-0)$ luminosity estimated in this work.

Finally, the dynamical mass of a galaxy also includes the mass of the non baryonic DM component, which is a source of uncertainty, as no independent measurement of this mass fraction is available for our SMGs. Unlike observations in local disk galaxies, which have reported DM fractions $\left(f_{\mathrm{DM}}\right)$ of $\sim 50 \%$ (e.g., Courteau \& Dutton 2015), it has been claimed by recent spectroscopic surveys (e.g., Price et al. 2016; Wuyts et al. 2016; Genzel et al. 2017) that more compact star-forming disk galaxies at $z>2$ appear to be heavily baryon dominated with $f_{\mathrm{DM}} \sim 10 \%-20 \%$, although these calculations involve the same unknown factors as those that are used for gas fractions. In contrast, recent simulations (e.g., Lovell et al. 2018) have reported that the DM fractions in disc-like galaxies could range up to $65 \%$ for galaxies with stellar masses similar to SMGs $\left(\sim 10^{11} M_{\odot}\right)$.

Combining Equations (2) and (3), the total mass can be expressed as:

$$
M_{\mathrm{dyn}}=\frac{L_{H} \times\left(M_{*} / L_{H}\right)+L_{\mathrm{CO}} \times \alpha_{\mathrm{CO}}}{\left(1-f_{\mathrm{DM}}\right)},
$$

where the conversion factor $\alpha_{\mathrm{CO}}$, the stellar mass-to-light ratio $\left(M_{*} / L_{H}\right)$, and the DM fraction $f_{\mathrm{DM}}$ are the unknown parameters. Given the small number of independent data points available (one for each of our four sources), in order to reduce the parameter space we will keep the $f_{\mathrm{DM}}$ value fixed, and discuss the effect of low and high $f_{\mathrm{DM}}$ values to the other parameters distributions later on.

First, we explore the parameter space built up in Equation (4) by applying an MCMC technique, using the open-source algorithm EMCEE (Foreman-Mackey et al. 2013) for the sampling. Based on the likelihood of the measured dynamical masses $M_{\text {dyn }}$ (estimated in Section 3.3 as a function of $L_{\mathrm{CO}}$ and $L_{H}$ ) given in this model, we sample the posterior probability density function (posterior PDF) for $\alpha_{\mathrm{CO}}$ and $\left(M_{*} / L_{H}\right)$. It is important to note that at this point the likelihood function accounts only for the uncertainties in the dynamical masses $M_{\text {dyn }}$. To account for the uncertainties in the $L_{\mathrm{CO}}$ and $L_{H}$ variables as well, we use a Monte Carlo approach and repeat the inference exercise presented above for 100 iterations, each time using values randomly chosen from within a normal distribution that corresponds to the mean and standard deviation of our $L_{\mathrm{CO}}$ and $L_{H}$ measurements. ${ }^{19}$ Finally, we average the posterior PDFs gathered in these 100 iterations and obtain a final probability distribution for the parameters that takes into account the uncertainties in the measurements of all observables $\left(M_{\mathrm{dyn}}, L_{\mathrm{CO}}\right.$ and $\left.L_{H}\right)$.

Figure 5 shows the one- and two-dimensional final posterior PDFs of the $\left(M_{*} / L_{H}\right)$ and $\alpha_{\mathrm{CO}}$ parameters adopting three different DM fractions $f_{\mathrm{DM}}=0 \%, 15 \%$, and $30 \%$. The covariance between

\footnotetext{
${ }^{19}$ We note that while $L_{H}$ is an independent measurement, $M_{\mathrm{dyn}}$ and $L_{\mathrm{CO}}$ are both a function of the velocity FWHM of the line emission $\Delta V_{\mathrm{FWHM}}$, which can affect the robustness of the parameter uncertainties inferred through the MCMC routine. In order to account for the correlated errors induced through this dependence while simulating the uncertainties of $L_{\mathrm{CO}}$, each Monte Carlo iteration adopts $M_{\mathrm{dyn}}\left(\Delta V_{\mathrm{FWHM}}\right)$ and $L_{\mathrm{CO}}\left(\Delta V_{\mathrm{FWHM}}\right)$, where $\Delta V_{\mathrm{FWHM}}$ assumes a single value for both functions and is each time randomly sampled from within a normal distribution that corresponds to the mean and standard deviation of $\Delta V_{\text {FWHM }}$. In this way, the correlated measurements of $M_{\text {dyn }}$ and $L_{\mathrm{CO}}$ are robustly accounted for during the Monte Carlo sampling.
}
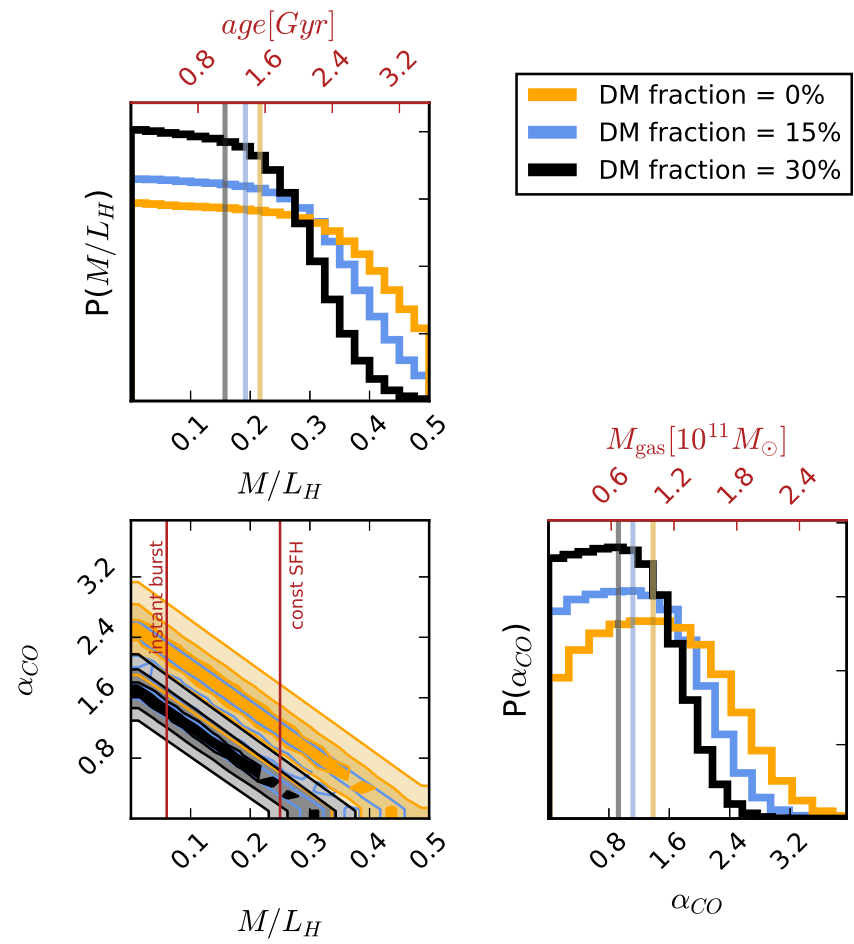

Figure 5. One- and two-dimensional posterior probability density functions (PDFs) of the $M_{*} / L_{H}$ and $\alpha_{\mathrm{CO}}$ parameters for the ALESS sources in this work. The upper and lower-right panels show the one-dimensional PDFs as histograms, where the orange, blue, and black solid lines correspond to the inference assuming dark matter contributions of $0 \%, 15 \%$, and $30 \%$, respectively. The galaxy age axis (upper panel, in red) equivalent to the $M_{*} / L_{H}$ axis is computed using the relation presented by Hainline et al. (2011) for an instantaneous burst SFH. The $M_{\text {gas }}$ axis (lower-right panel, in red) is computed by adopting the median $L_{\mathrm{CO}}$ of our sample, $\left\langle L_{\mathrm{CO}}\right\rangle=7.7 \times$ $10^{10} \mathrm{~K} \mathrm{~km} \mathrm{~s}^{-1} \mathrm{pc}^{-2}$. The transparent orange, blue, and black vertical lines correspond to the median values of the distributions. The lower-left panel shows the covariance plot (two-dimensional PDF) of the parameters, where a clear correlation can be recognized through the diagonal shape of the contours, which represent the $25 \mathrm{th}, 50 \mathrm{th}$, and 75 th percentiles of the sampled distribution. Two reference lines (red) are drawn, corresponding to the average $M_{*} / L_{H}$ ratio found by Simpson et al. (2014) for the ALESS survey as a whole, assuming a constant SFH in contrast to an instantaneous burst SFH.

the parameters can be recognized in the two-dimensional PDF (Figure 5) as an elongated ellipse in the central contour level plot. Higher DM fractions decrease the value of $\alpha_{\mathrm{CO}}$ and consequently $M_{\text {gas }}$. This trend is similarly followed by the $\left(M_{*} / L_{H}\right)$ parameter. For a DM fraction of $15 \%$ we find a median value of $\alpha_{\mathrm{CO}}=1.1_{-0.7}^{+0.8}\left[M_{\odot} /\left(\mathrm{K} \mathrm{km} \mathrm{s}^{-1} \mathrm{pc}^{2}\right)^{-1}\right]$, while for a larger DM fraction of $30 \%$, these values decrease to $\alpha_{\mathrm{CO}}=$ $0.9_{-0.6}^{+0.7}\left[M_{\odot} /\left(\mathrm{K} \mathrm{km} \mathrm{s}^{-1} \mathrm{pc}^{2}\right)^{-1}\right]$. For $f_{\mathrm{DM}}=0$ we obtain an upper limit to $\alpha_{\mathrm{CO}} \leqslant 1.4_{-0.9}^{+0.9}\left[M_{\odot} /\left(\mathrm{K} \mathrm{km} \mathrm{s}^{-1} p c^{2}\right)^{-1}\right]$. For the massto-light ratio parameter we find $\left(M_{*} / L_{\mathrm{H}}\right)=0.22_{-0.15}^{+0.16} M_{\odot} / L_{\odot}$, $\left(M_{*} / L_{\mathrm{H}}\right)=0.19_{-0.13}^{+0.15} M_{\odot} / L_{\odot}$, and $\left(M_{*} / L_{\mathrm{H}}\right)=0.16_{-0.10}^{+0.12} M_{\odot} / L_{\odot}$ for DM fractions of $0 \%, 15 \%$, and $30 \%$. Using these values, the gas reservoirs in our sources have an average gas mass of $\sim 0.9 \times 10^{11} M_{\odot}(\mathrm{DM}$ contribution of $15 \%)$ and $\sim 0.7 \times 10^{11} M_{\odot}$ (DM contribution of 30\%), corresponding to average gas-to-totalmass fractions of $0.26 \%$ and $0.33 \%$, respectively. These sources show similar gas fractions than those reported for typical starforming galaxies $(\leqslant 50 \%$, Tacconi et al. 2017). For comparison, Bothwell et al. (2013) presented a study of a representative sample of SMGs at $z \sim 2$ from predominantly $\mathrm{CO}(3-2)$ observations, finding mean gas masses of $(3.2 \pm 2.1) \times 10^{10} M_{\odot}$ assuming $\alpha_{\mathrm{CO}}=1$. Since the resulting PDF for the $M_{*} / L_{H}$ and age 
parameters for our galaxies is broad and poorly constrained, the galaxy stellar population properties inferred through this method ( 0.1-2.0 Gyr) easily agree with those estimated through SED fitting by da Cunha et al. (2015), which are also associated with large errors $(\sim 70 \%)$. They find the age of these galaxies range between $\sim 0.04$ and $1.22 \mathrm{Gyr}$, corresponding to $M_{*} / L_{H}$ between 0.16 and 0.2 .

As discussed above, the values recovered for the parameters are subject to large uncertainties due to our small sample size. However, we note that one goal of this exercise is to shed light upon the degeneracies among the key parameters and large uncertainties inherent to gas mass calculations. Most importantly, given the increasing availability of dynamical mass measurements for high-redshift galaxies with ALMA, we consider that this approach has potential for constraining the molecular gas properties with more accuracy, especially since larger sample sizes will allow the exploration of a more detailed parameter space in the future.

\section{Distributions of Molecular Gas, Dust Continuum and Stellar Emission}

\subsection{Offset Distributions of ISM Tracers and Stellar Emission in ALESS122.1}

An interesting case among our samples is the apparently uncorrelated distribution of dust continuum, gas, and stars in ALESS122.1 (Figure 6). The gas and stellar emission extend across regions of similar size, separated by $\sim 0 . .5(\sim 5 \mathrm{kpc}$ at $z=2$ ) as measured from their centroids, but are tightly aligned next to each other. There is almost no overlap of gas and stellar emission, even when considering the combined astrometric uncertainties $^{20}(<0$ "! 1$)$. Moreover, while the gas and stars have half-light radii $r_{1 / 2} \sim 4 \mathrm{kpc}$ and are thus extended on scales of $\sim 10 \mathrm{kpc}$, the detected dust continuum emission $(3 \mathrm{~mm}$ observed-frame, corresponding to $\sim 850 \mu \mathrm{m}$ rest-frame) is confined to a central region of $\sim 5 \mathrm{kpc}$, with the emission peak spatially coincident with that of the molecular gas emission. Similar physical offsets between the dust continuum, gas, and stellar emission, and specifically between ALMA and HST, have been previously found in a number of high-redshift sources (e.g., Riechers et al. 2010; Chen et al. 2015; Hodge et al. 2015; Elbaz et al. 2017; Fujimoto et al. 2017; Simpson et al. 2017). For example, based on low-resolution $870 \mu \mathrm{m}$ continuum data $(\sim 1 . \prime 5)$, Chen et al. (2015) reported a statistical offset with the existing stellar component traced by $H S T$ as large as $\Delta p=0 . " 4(\sim 4 \mathrm{kpc}$ at $z=2)$ in $66 \%$ of the ALESS SMGs. Similarly, Hodge et al. (2015) found in the $z=4$ galaxy, GN20, that the FIR and CO emission are offset by 0!" 6 $(4 \mathrm{kpc})$ from the peak of the rest-frame UV emission as traced by the $H S T / \mathrm{WFC} 3 \mathrm{~F} 105 \mathrm{~W}$ image.

We consider three scenarios for explaining the mismatch of the distributions of stellar and gas/dust continuum emission in ALESS122.1. The first and most plausible scenario assumes that the optical component extends to the regions of gas and dust continuum emission, while suffering extreme extinction in these regions. Such high-extinction scenarios have been suggested previously in starbursts with extreme SFRs and in cases of partial or total absence of optical counterparts (e.g., Hodge et al. 2012; Walter et al. 2012; Simpson et al. 2015). This is not uncommon in submm selected samples, where

\footnotetext{
${ }^{20}$ The HST astrometric uncertainties are low since the image has been accurately calibrated using GAIA measurements of this field.
}

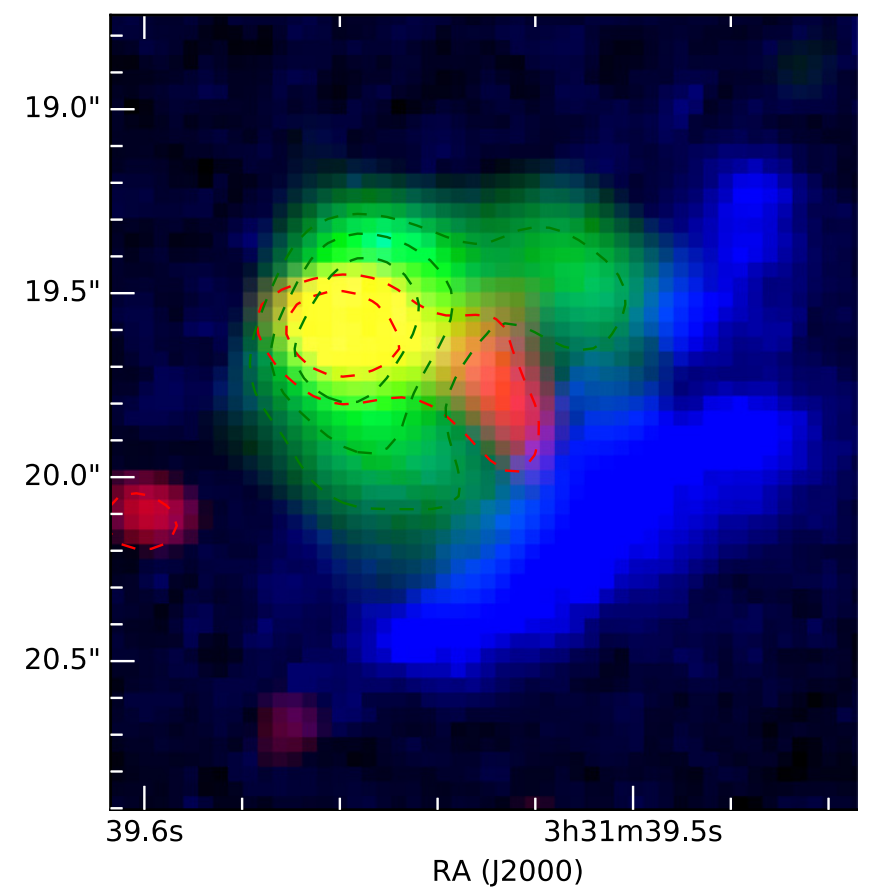

Figure 6. False-color image of the molecular gas, dust continuum, and stellar emission components in ALESS122.1. The molecular gas (green) shows the $\mathrm{CO}(3-2)$ emission and the dust continuum region (red) corresponds to the $3 \mathrm{~mm}$ (rest-frame $\sim 850 \mu \mathrm{m}$ ) continuum detection $(4 \sigma)$, both masked at $S \geqslant 3 \sigma$. For clarity we overplot dashed contours for both the CO $(6,8,10 \sigma)$ and dust continuum emission ( 3 and $4 \sigma$ ), to show that their centroids are colocated. The stellar emission (HST-ACS F814W) is presented as the blue region and shows a large offset from the gas and dust centroid, with almost no overlap even when considering the combined astrometric uncertainties. The HST astrometry in this data is accurate since the images were corrected based on GAIA measurements. The increasing evidence of a population of sources with misaligned stellar, dust continuum, and gas emission has important implications for energy balance assumptions in SED fitting. We discuss the striking difference between the observed extent of the gas and dust continuum emission for a larger sample of sources in Section 4.2.

optical detection rates are only $\sim 70 \%-80 \%$ even with deep data (e.g., Simpson et al. 2014).

The second scenario implies that the observed offset reflects a physical misalignment between the gas/dust continuum distributions and that of the stellar mass. This argument has been tested by Chen et al. (2015) for the full ALESS sample. They tested this hypothesis by comparing the positional offsets found in low-redshift $(z<2)$ to those in high redshift $(z>2)$ subsamples, taking into account that at lower redshift the optical photometry would probe wavelengths less affected by obscuration. They found no statistical difference in the measured positional scatter between the low- and high-redshift samples, implying that obscuration is not the dominant factor for the population as a whole.

The third scenario implies that ALESS122.1 is an ongoing, merging system comprised of two components, one of which is a heavily obscured SMG that has no detected optical counterpart, and the other is an optically bright galaxy in the HST-ACS F814W imaging (which corresponds to rest-frame $\sim 3000 \AA$ ). High-resolution optical-MIR counterparts at other wavelengths are needed in order to confirm the nature of this source and explain the uncorrelated distributions of its physical components. 
Regardless of the underlying scenario, the significant anticorrelation between the dust continuum and optical imaging disprove some assumptions that are intrinsic to multiwavelength studies such as energy balance in SED fitting (this has been previously discussed by e.g., Hainline et al. 2011; Chen et al. 2015, 2017; Hodge et al. 2016; Simpson et al. 2017).

\subsection{Statistical Analysis on the Relative Sizes of Dust Continuum, Molecular Gas and Stellar Emission in SMGs}

To gain a general understanding of the distributions of the molecular gas, dust continuum, and stellar emission in SMGs and how they relate to each other, next we conduct a stacking analysis using the $\mathrm{CO}$ emission measured in our sources and the available multiwavelength data at similar resolution.

We first produced the stacked radial profiles of the $\mathrm{CO}$ emission of the four ALESS sources presented here. To achieve this, we create $10 \times 10^{\prime \prime}$ thumbnails of our sources, align their centers and scale them to have the same peak flux. We then produce the radial profiles of the individual sources by radially averaging the emission centered at the source peak, and finally, we average the individual radial profiles in our sample (Figure 7). ${ }^{21}$ To account for the effect of outlier structures of the individual sources, we estimate the uncertainties of the radial profiles through bootstrapping, e.g., by creating 1000 different average profiles, where each iteration used a randomly resampled combination of sources.

Using the same technique, we also stack high-resolution dust continuum emission ( 0 !' 16 FWHM at rest-frame $250 \mu \mathrm{m}$ ) of 16 luminous ALESS sources studied by Hodge et al. (2016). This sample of sources is representative of the ALESS survey as a whole (ALESS median $z=2.5 \pm 0.2$ and $L_{\mathrm{IR}}=(3.0 \pm$ $0.3) \times 10^{12} L_{\odot}$, Simpson et al. 2014; Swinbank et al. 2014) as it has a median redshift of $z=2.6 \pm 0.5$ and infrared luminosity of $L_{\mathrm{IR}}=(3.6 \pm 0.9) \times 10^{12} L_{\odot}$. Most importantly, these properties are also comparable to those of the sample used for the $\mathrm{CO}$ stacked profiles (median redshift of $z=2.5 \pm 0.4$ and infrared luminosity of $L_{\mathrm{IR}}=(5.6 \pm 2.2) \times$ $\left.10^{12} L_{\odot}\right)$, although both samples have only one source in common (ALESS67.1).

Finally, we stack high-resolution HST imaging ( 0 "! 17 in the $H_{160}$ band) available for the same 16 ALESS sources following the procedure described above. The stacked surface brightness profiles for the gas, dust continuum, and stellar components are shown in Figure 7 as green, red, and blue data points, respectively.

To quantify the extent and distribution of the molecular, submm and optical/near-infrared emission, first we assume that the observed emission can be described by an exponential profile (Sérsic profile with index $n=1$ ) convolved with a Gaussian profile, which corrects for the effect of the shape of the beam. Based on this we fit a one-dimensional exponential to the $\mathrm{CO}$ and dust continuum profiles and find that they are well described by such a model. We note that for fitting the dust profile we discarded the data points at radius of $>7 \mathrm{kpc}$, since these are evidently affected by artifacts produced by the side lobes of the beam (shown as the shaded area in Figure 7). ${ }^{22}$

\footnotetext{
${ }^{21}$ We would like to note that we investigated the difference between the approach described above and an approach in which a stacked image is produced first and the mean radial profile is then extracted directly from the stacked image. We find that both methods are equivalent and result in identical average radial profiles.

22 This issue is not relevant for $\mathrm{CO}$, since the side lobes appear only at $r>15 \mathrm{kpc}$.
}

The radial profile of the stellar emission results in a bad quality fit when using an exponential, i.e., when fixing the Sérsic index $n=1$. To improve this, we leave the Sérsic index as a free parameter and achieve a better quality fit with $n=0.8 \pm 0.2$, which is consistent within the errors with the median value found for the ALESS sample as whole $(n=1.2 \pm 0.3$, Chen et al. 2015). The best-fit models consist then of exponential profiles with radii of $r=1.7 \pm 0.1, \quad 3.8 \pm 0.1$, and $4.0 \pm 2.0 \mathrm{kpc}$ for the dust continuum, $\mathrm{CO}$, and stellar emission, respectively.

These results reveal that both the stellar $\left(H_{160}\right)$ and molecular gas $(\mathrm{CO}(J=3-2))$ emission have similar sizes, but are $>2 \times$ more extended than the dust continuum emission, measured at rest-frame $250 \mu \mathrm{m}$. In addition, we note that the estimated size of the $\mathrm{CO}(3-2)$ emission should be considered a lower limit for the extent of the total molecular-mass distribution, as the ground state transition $\mathrm{CO}(1-0)$ has been observed to be even more extended than the higher $J$-transitions (Engel et al. 2010).

\subsection{Physical Implications of the More Extended Molecular Gas Distributions as Compared to Dust Continuum Emission}

A crucial consequence of the different scales probed by the molecular gas, dust, and stellar tracers in these galaxies (as shown in Section 4.2) is the uncertainty in using conversion factors between the luminosities of individual tracers. The compactness of the dust continuum emission in SMGs has been discussed in the literature (Ikarashi et al. 2015; Simpson et al. 2015; Barro et al. 2016; Hodge et al. 2016; Simpson et al. 2017; Tadaki et al. 2017a), as ALMA continuum observations at high angular resolution $(0$ ". $13-0$." 4) have revealed median radii of $\sim 0.7-1.5 \mathrm{kpc}$.

Apart from $\mathrm{CO}$ and optical imaging, high-resolution observations of other SF tracers such as synchrotron and free-free emission have allowed a comparison of relative sizes of dust continuum and gas emission regions. Indeed, radio interferometric observations of SMGs with Very Large Array at 1.4 GHz (Chapman et al. 2004; Biggs \& Ivison 2008), at $3 \mathrm{GHz}$ (Miettinen et al. 2015, 2017), and at $6 \mathrm{GHz}$ have shown that dust continuum sizes appear to be 1.4-4.4 times smaller than the ratio.

What is the origin of the significantly smaller sizes $(>2)$ of the dust continuum emission in SMGs compared to the other physical components? There are three parameters that could affect the emission of dust at larger radii: the dust-to-gas ratio, the dust temperature, and the optical depth of the dust and gas. The small extent of the dust continuum emission at a rest-frame $250 \mu \mathrm{m}$ is likely to trace the heating of dust by SF or AGN, as it is confined to central compact regions, as expected for highly star-forming systems. A decrease in optical depth at larger radii would similarly affect the observed submm continuum emission, as would a radially dependent dust-to-gas ratio. Studies in the nearby universe, however, (e.g., Sandstrom et al. 2013; Groves et al. 2015) suggest that no large variations in the latter are expected across the galaxy, making this scenario less plausible.

The different spacial extent for the rest-frame $250 \mu \mathrm{m}$ dust continuum emission and the CO line emission can be explained in the context of self-consistent radiative transfer of the $\mathrm{CO}$ (3-2) and dust continuum emission. Weiß et al. (2007) presented such a model, where the line and dust continuum emission are linked through a constant dust-to-gas mass ratio throughout the galaxy, specifically, by connecting the gas 


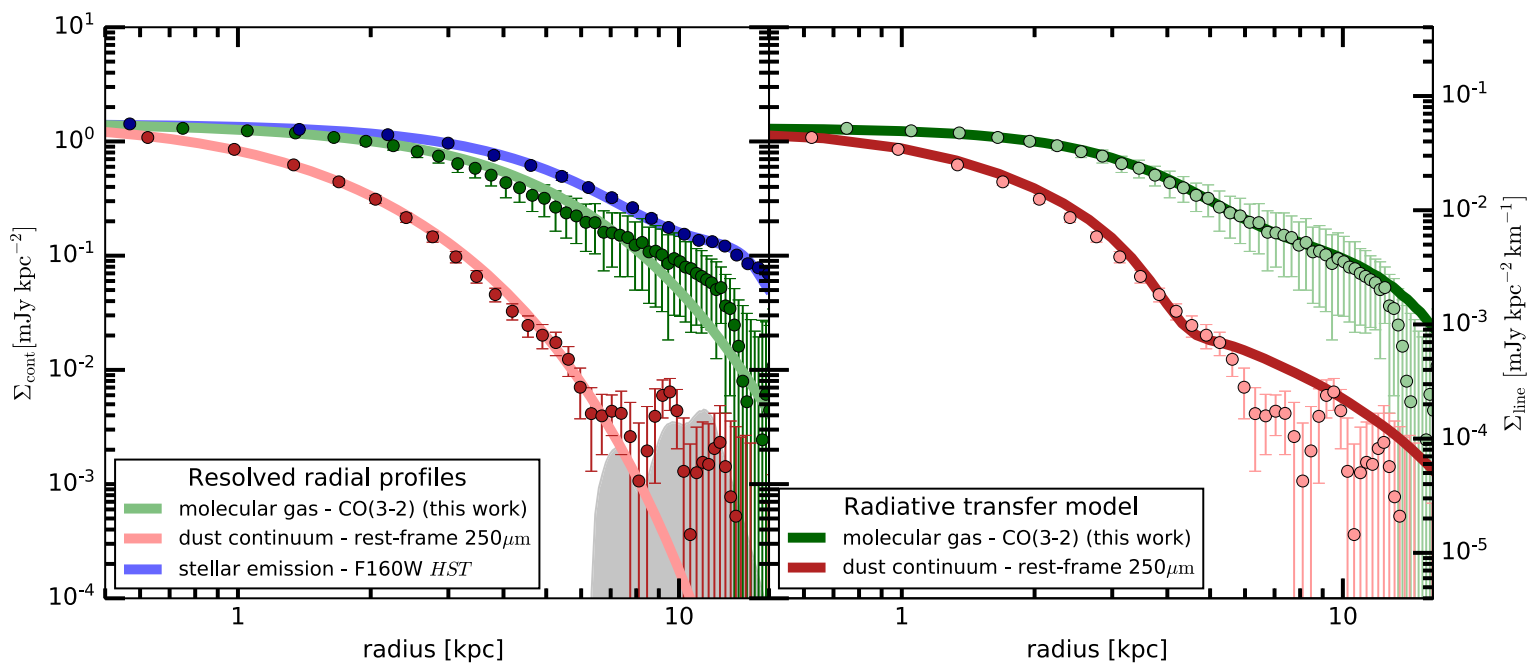

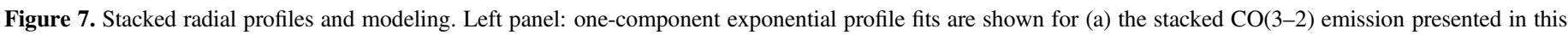

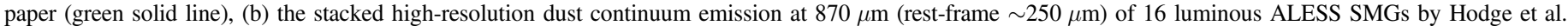

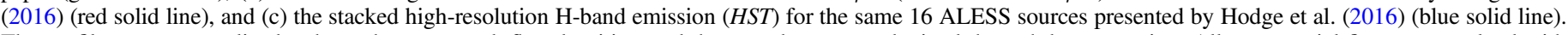

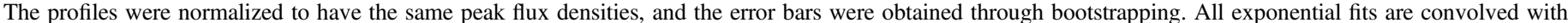

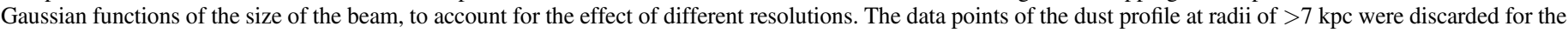

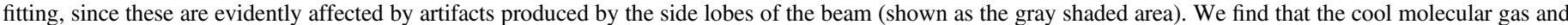

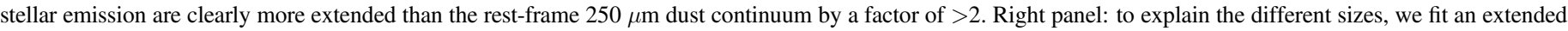

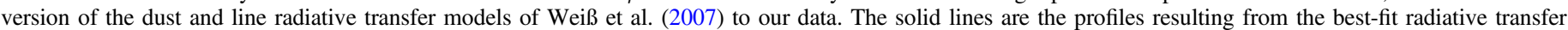

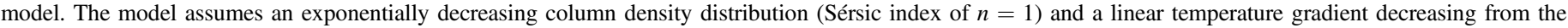

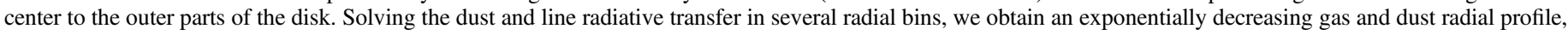

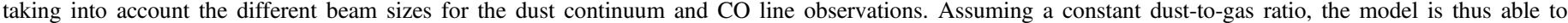
reproduce the apparent size differences between the $\mathrm{CO}(3-2)$ and dust continuum emission by only introducing temperature and optical depth gradients.

column density $N_{\mathrm{H}_{2}}$ to the optical depth of the dust, $\tau_{\text {dust }}(r) \sim N_{\text {dust }} \sim N_{\mathrm{H}_{2}}$. The different dust continuum and $\mathrm{CO}$ sizes can then be explained by assuming a radially decreasing gas column density and a radially decreasing temperature distribution $T(r)$. Following the radiative transfer equation, and neglecting the background temperature, we have

$$
S(r) \propto T(r) \cdot\left(1-e^{-\tau}\right),
$$

where $T(r)$ implies $T(r)=T_{\text {dust }}(r)$ for the dust and $T(r)=T_{\mathrm{ex}}(r)$ for the molecular gas. We can expect that the long wavelength dust continuum emission, which is optically thin $(\tau \ll 0)$, will be sensitive to both the column density and the temperature gradients $\left(S_{\text {cont }}(r) \propto T_{\text {dust }}(r) \cdot \tau_{\text {dust }}(r)\right)$, whereas the optically thick CO line emission $(\tau \gg 1)$ will to first order only respond to the temperature gradient $\left(S_{\mathrm{CO}}(r) \propto T_{\mathrm{ex}}(r) \sim T_{\text {kin }}(r)\right.$ for the low-J CO transitions, which are close to thermalization. This would have the effect that the dust continuum emission becomes fainter more rapidly, as a function of radius, than the gas emission.

Based on these arguments, we have used an extended version of the dust and line radiative transfer models of Weiß et al. (2007) to demonstrate this effect for the radial distribution of the stacked $\mathrm{CO}(3-2)$ and rest-frame $250 \mu \mathrm{m}$ dust continuum data shown in Figure 7. In the model we use an exponentially decreasing column density distribution (Sérsic index of $n=1$ ) and a linear temperature gradient from the center to the outer parts of the disk, and we solve the dust and line radiative transfer in several radial bins (more details on the model will be presented in A. Weiß et al. 2018, in preparation). Figure 7 shows the fit of an exponentially decreasing gas component, taking into account the different beam sizes for the dust continuum and CO line observations. Given the uncertainties of the data, the model can reproduce the different radial behavior of the dust continuum and the $\mathrm{CO}(3-2)$ line emission. From this fit we derive an intrinsic exponential scale length of $3.4 \mathrm{kpc}$ $(0$ ". 4$)$ for the underlying $\mathrm{H}_{2}$ distribution, intermediate between the intrinsic sizes we have estimated earlier based on the deconvolved intensity profiles for the dust and the $\mathrm{CO}(3-2)$ line emission.

In this section we have proposed that assuming a unique intrinsic distribution of dust and gas, temperature, and optical depth gradients alone could give rise to the apparent size differences observed between the $\mathrm{CO}$ and dust continuum emission. If these parameters are the origin of the compactness of dust continuum emission observed in the high-redshift universe (here probed at a rest-frame $\sim 250 \mu \mathrm{m}$ ), caution must be exercised when extrapolating properties of high-resolution continuum observations to conclusions on the molecular phase of the ISM. Specifically, these results advise against combining unresolved molecular gas observations with radius estimates from resolved dust continuum observations when calculating key parameters such as dynamical masses. In order to empirically test the assumptions placed into this model on the temperature and optical depth gradients, high-resolution observations of the dust continuum at different frequencies would be necessary, as a gradient in observed sizes of the emission regions is a direct tracer of the dust optical depth across the SED.

\section{Summary}

1. We have presented ALMA observations of the gas and dust of four luminous submillimeter galaxies at $z \sim 2-3$ to investigate the spatially resolved properties of the ISM on scales of a few kpc. The molecular gas in these sources, traced by the ${ }^{12} \mathrm{CO}(J=3-2)$ emission, is extended over FWHM $\sim 5-14$ kpc. 
2. We investigated the dynamics of the molecular gas in our sources and modeled the kinematics of one of them, ALESS122.1, finding that the velocity fields of three of our four sources are consistent with disk rotation to the first order. We clarify that this scenario does not preclude the connection of our sources to mergers as observations of a nearby merger remnant and simulations show that gas disks reform rapidly within $>100$ Myr of the peak SF associated with a merger.

3. The resolved $\mathrm{CO}$ imaging provides size measurements that allow us to derive dynamical masses for our sample. The dynamical masses found are in the range of (1.1-5.3) $\times 10^{11} M_{\odot}$, as calculated within $2 \times$ the halflight radii of our sources, which is in agreement with other dynamical mass estimates for SMGs.

4. To provide dynamical constraints to the gas masses, we explore the uncertainties introduced by the estimation of stellar masses, the assumptions on the DM fractions and the $\mathrm{CO}-$ to- $\mathrm{H}_{2}$ conversion factor $\alpha_{\mathrm{CO}}$. Taking into account the covariance between the mass-to-light ratio and $\alpha_{\mathrm{CO}}$ parameters, we estimate an average $\mathrm{CO}$-to- $\mathrm{H}_{2}$ conversion factor of $\alpha_{\mathrm{CO}}=1.1_{-0.7}^{+0.8}$ and $\alpha_{\mathrm{CO}}=0.9_{-0.6}^{+0.7}$ for $\mathrm{DM}$ fractions of $15 \%$ and $30 \%$, respectively, and an upper limit of $\alpha_{\mathrm{CO}} \leqslant 1.4_{-0.9}^{+0.9}$ for DM fractions of $0 \%$. These values imply gas fractions of $\sim 30 \%$ for our sources, which are similar to those estimated for main-sequence star-forming galaxies and other SMGs.

5. Our high-resolution study allows us to investigate the correlation between the spatial distribution of the physical components of the ISM (the dust continuum and gas) and the stellar distributions. The sizes of gas and stars are comparable but spatially uncorrelated, while the restframe $250 \mu \mathrm{m}$ dust continuum is significantly more compact. The observation of the anti-correlated distributions of the dust continuum and gas emission with respect to the unobscured stellar emission may challenge energy balance assumptions in global SED fitting routines and suggests that caution must be exercised, particularly for dusty star-forming galaxies, such as SMGs.

6. To investigate this question statistically, we conduct a stacking analysis of available high-resolution ancillary data for SMG populations of similar properties. We find that the cool molecular gas and stellar emission are clearly more extended than the rest-frame $250 \mu \mathrm{m}$ dust continuum by a factor of $>2$.

7. We reproduce our observations with a radiative transfer model, finding that the different sizes are consistent with the expected response of optically thin dust and optically thick gas to radially decreasing optical depth and temperature gradients, when a constant dust-to-gas ratio is assumed. We suggest that extrapolations from the morphological properties of high-resolution continuum observations to conclusions on the molecular phase of the ISM should be thus treated cautiously.

We thank the anonymous referee for their useful comments to improve the clarity of this manuscript. We would like to thank Jarle Brinchmann, Erwin de Blok, Alison Peck and the Allegro group in Leiden, especially Luke Maud, for valuable help and advice at different stages of the project. G.C.R. acknowledges support from the European Research Council under the European Unions Seventh Framework Programme (FP/2007-2013)/ERC Advanced Grant NEW-CLUSTERS321271. J.H. acknowledges support of the VIDI research programme with project number 639.042.611, which is (partly) financed by the Netherlands Organization for Scientific Research (NWO). I.R.S. acknowledges support from the ERC Advanced Grant DUSTYGAL (321334), a Royal Society/ Wolfson Merit Award and STFC (ST/P000541/1). J.L.W. acknowledges support of an STFC Ernest Rutherford Fellowship (ST/P004784/1) and additional support from STFC (ST/ P000541/1). F.W. and B.P.V. acknowledge funding through ERC grants "Cosmic Dawn" and "Cosmic Gas." H.D. acknowledges financial support from the Spanish Ministry of Economy and Competitiveness (MINECO) under the 2014 Ramón y Cajal program MINECO RYC-2014-15686. This paper makes use of the following ALMA data: ADS/JAO. ALMA\#2013.1.00470.S and \#2016.1.00754.S. ALMA is a partnership of ESO (representing its member states), NSF (USA) and NINS (Japan), together with NRC (Canada), MOST and ASIAA (Taiwan), and KASI (Republic of Korea), in cooperation with the Republic of Chile. The Joint ALMA Observatory is operated by ESO, AUI/NRAO and NAOJ. This research made use of ASTROPY, a community-developed core Python package for Astronomy (Astropy Collaboration et al. 2013), and the open-source plotting packages for Python APLPY (Robitaille \& Bressert 2012), and CORNER (ForemanMackey 2016).

\section{ORCID iDs}

Gabriela Calistro Rivera (i) https://orcid.org/0000-00030085-6346

J. A. Hodge (1) https://orcid.org/0000-0001-6586-8845 Ian Smail (i) https://orcid.org/0000-0003-3037-257X A. M. Swinbank (i) https://orcid.org/0000-0003-1192-5837

A. Weiss (iD https://orcid.org/0000-0003-4678-3939

J. L. Wardlow (iD https://orcid.org/0000-0003-2376-8971

F. Walter (i) https://orcid.org/0000-0003-4793-7880

Chian-Chou Chen (iD https://orcid.org/0000-0002-3805-0789

W. N. Brandt (iD https://orcid.org/0000-0002-0167-2453

K. Coppin (iD https://orcid.org/0000-0002-0729-2988

H. Dannerbauer (i) https://orcid.org/0000-0001-7147-3575

T. R. Greve (i) https://orcid.org/0000-0002-2554-1837

A. Karim (1D https://orcid.org/0000-0002-8414-9579

K. K. Knudsen (i) https://orcid.org/0000-0002-7821-8873

E. Schinnerer (iD https://orcid.org/0000-0002-3933-7677

J. M. Simpson (1) https://orcid.org/0000-0002-8521-1995

B. Venemans (1D https://orcid.org/0000-0001-9024-8322

\section{References}

Accurso, G., Saintonge, A., Catinella, B., et al. 2017, MNRAS, 470, 4750 Aravena, M., Hodge, J. A., Wagg, J., et al. 2014, MNRAS, 442, 558

Astropy Collaboration, Robitaille, T. P., Tollerud, E. J., et al. 2013, A\&A, 558, A33

Barro, G., Kriek, M., Pérez-González, P. G., et al. 2016, ApJL, 827, L32

Biggs, A. D., \& Ivison, R. J. 2008, MNRAS, 385, 893

Blain, A. W., Smail, I., Ivison, R. J., Kneib, J.-P., \& Frayer, D. T. 2002, PhR, 369,111

Bolatto, A. D., Wolfire, M., \& Leroy, A. K. 2013, ARA\&A, 51, 207

Bothwell, M. S., Smail, I., Chapman, S. C., et al. 2013, MNRAS, 429, 3047

Bouché, N., Carfantan, H., Schroetter, I., Michel-Dansac, L., \& Contini, T. 2015, AJ, 150, 92

Carilli, C. L., \& Walter, F. 2013, ARA\&A, 51, 105

Casey, C. M., Narayanan, D., \& Cooray, A. 2014, PhR, 541, 45 
Chapman, S. C., Smail, I., Windhorst, R., Muxlow, T., \& Ivison, R. J. 2004, ApJ, 611, 732

Chen, C.-C., Hodge, J. A., Smail, I., et al. 2017, ApJ, 846, 108

Chen, C.-C., Smail, I., Swinbank, A. M., et al. 2015, ApJ, 799, 194

Courteau, S., \& Dutton, A. A. 2015, ApJL, 801, L20

da Cunha, E., Walter, F., Smail, I. R., et al. 2015, ApJ, 806, 110

Danielson, A. L. R., Swinbank, A. M., Smail, I., et al. 2011, MNRAS, 410, 1687

Danielson, A. L. R., Swinbank, A. M., Smail, I., et al. 2017, ApJ, 840, 78

Dannerbauer, H., Lehnert, M. D., Emonts, B., et al. 2017, A\&A, 608, A48

de Blok, W. J. G., \& Walter, F. 2014, AJ, 147, 96

Decarli, R., Walter, F., Aravena, M., et al. 2016, ApJ, 833, 70

Elbaz, D., Leiton, R., Nagar, N., et al. 2017, arXiv:1711.10047

Emonts, B. H. C., Lehnert, M. D., Villar-Martín, M., et al. 2016, Sci, 354, 1128

Engel, H., Tacconi, L. J., Davies, R. I., et al. 2010, ApJ, 724, 233

Erb, D. K., Steidel, C. C., Shapley, A. E., et al. 2006, ApJ, 647, 128

Foreman-Mackey, D. 2016, JOSS, 1, 24

Foreman-Mackey, D., Hogg, D. W., Lang, D., \& Goodman, J. 2013, PASP, 125,306

Fujimoto, S., Ouchi, M., Shibuya, T., \& Nagai, H. 2017, ApJ, 850, 83

Gaia Collaboration, Brown, A. G. A., Vallenari, A., et al. 2016, A\&A, 595, A2

Genzel, R., Schreiber, N. M. F., Übler, H., et al. 2017, Natur, 543, 397

Genzel, R., Tacconi, L. J., Combes, F., et al. 2012, ApJ, 746, 69

Genzel, R., Tacconi, L. J., Lutz, D., et al. 2015, ApJ, 800, 20

Ginolfi, M., Maiolino, R., Nagao, T., et al. 2017, MNRAS, 468, 3468

Greve, T. R., Vieira, J. D., Weiß, A., et al. 2012, ApJ, 756, 101

Groves, B. A., Schinnerer, E., Leroy, A., et al. 2015, ApJ, 799, 96

Hainline, L. J., Blain, A. W., Smail, I., et al. 2011, ApJ, 740, 96

Harris, A. I., Baker, A. J., Frayer, D. T., et al. 2012, ApJ, 752, 152

Hezaveh, Y. D., Marrone, D. P., \& Holder, G. P. 2012, ApJ, 761, 20

Hildebrand, R. H. 1983, QJRAS, 24, 267

Hodge, J. A., Carilli, C. L., Walter, F., et al. 2012, ApJ, 760, 11

Hodge, J. A., Carilli, C. L., Walter, F., Daddi, E., \& Riechers, D. 2013a, ApJ, 776,22

Hodge, J. A., Karim, A., Smail, I., et al. 2013b, ApJ, 768, 91

Hodge, J. A., Riechers, D., Decarli, R., et al. 2015, ApJL, 798, L18

Hodge, J. A., Swinbank, A. M., Simpson, J. M., et al. 2016, ApJ, 833, 103

Hopkins, P. F., Cox, T. J., Hernquist, L., et al. 2013, MNRAS, 430, 1901

Hopkins, P. F., Cox, T. J., Younger, J. D., \& Hernquist, L. 2009, ApJ, 691, 1168

Hubble, E. P. 1926, ApJ, 64, doi:10.1086/143018

Huynh, M. T., Emonts, B. H. C., Kimball, A. E., et al. 2017, MNRAS, 467, 1222

Ikarashi, S., Ivison, R. J., Caputi, K. I., et al. 2015, ApJ, 810, 133

Ivison, R. J., Papadopoulos, P. P., Smail, I., et al. 2011, MNRAS, 412, 1913

Ivison, R. J., Smail, I., Papadopoulos, P. P., et al. 2010, MNRAS, 404, 198

Karim, A., Swinbank, A. M., Hodge, J. A., et al. 2013, MNRAS, 432, 2

Lagos, C. d. P., Bayet, E., Baugh, C. M., et al. 2012, MNRAS, 426, 2142

Lehmer, B. D., Brandt, W. N., Alexander, D. M., et al. 2005, ApJS, 161, 21

Leroy, A. K., Bolatto, A., Gordon, K., et al. 2011, ApJ, 737, 12

Lovell, M. R., Pillepich, A., Genel, S., et al. 2018, arXiv:1801.10170

Madau, P., \& Dickinson, M. 2014, ARA\&A, 52, 415

Magdis, G. E., Daddi, E., Elbaz, D., et al. 2011, ApJL, 740, L15
McMullin, J. P., Waters, B., Schiebel, D., Young, W., \& Golap, K. 2007, in ASC Ser. 376, Astronomical Data Analysis Software and Systems XVI ed. R. A. Shaw, F. Hill, \& D. J. Bell (San Francisco, CA: ASP), 127

Michałowski, M. J., Dunlop, J. S., Cirasuolo, M., et al. 2012, A\&A, 541, A85

Miettinen, O., Novak, M., Smolčić, V., et al. 2015, A\&A, 584, A32

Miettinen, O., Novak, M., Smolčić, V., et al. 2017, A\&A, 602, A54

Narayanan, D., Krumholz, M., Ostriker, E. C., \& Hernquist, L. 2011, MNRAS, 418,664

Narayanan, D., \& Krumholz, M. R. 2014, MNRAS, 442, 1411

Narayanan, D., Krumholz, M. R., Ostriker, E. C., \& Hernquist, L. 2012, MNRAS, 421, 3127

Nedyalkov, P. L. 1993, AstL, 19, 115

Oteo, I., Ivison, R. J., Negrello, M., et al. 2017, arXiv:1709.04191

Planck Collaboration, Ade, P. A. R., Aghanim, N., et al. 2014, A\&A, 571, A16

Price, S. H., Kriek, M., Shapley, A. E., et al. 2016, ApJ, 819, 80

Riechers, D. A., Capak, P. L., Carilli, C. L., et al. 2010, ApJL, 720, L131

Riechers, D. A., Hodge, J., Walter, F., Carilli, C. L., \& Bertoldi, F. 2011, ApJL, 739, L31

Robertson, B., Bullock, J. S., Cox, T. J., et al. 2006, ApJ, 645, 986

Robitaille, T., \& Bressert, E. 2012, APLpy: Astronomical Plotting Library in Python, Astrophysics Source Code Library, ascl:1208.017

Sandstrom, K. M., Leroy, A. K., Walter, F., et al. 2013, ApJ, 777, 5

Scoville, N., Aussel, H., Sheth, K., et al. 2014, ApJ, 783, 84

Scoville, N., Lee, N., Vanden Bout, P., et al. 2017, ApJ, 837, 150

Scoville, N., Sheth, K., Aussel, H., et al. 2016, ApJ, 820, 83

Serjeant, S. 2012, MNRAS, 424, 2429

Sharon, C. E., Riechers, D. A., Hodge, J., et al. 2016, ApJ, 827, 18

Simpson, J. M., Smail, I., Swinbank, A. M., et al. 2015, ApJ, 799, 81

Simpson, J. M., Smail, I., Swinbank, A. M., et al. 2017, ApJ, 839, 58

Simpson, J. M., Swinbank, A. M., Smail, I., et al. 2014, ApJ, 788, 125

Solomon, P. M., \& Vanden Bout, P. A. 2005, ARA\&A, 43, 677

Sorba, R., \& Sawicki, M. 2018, MNRAS, arXiv:1801.07368

Spilker, J. S., Marrone, D. P., Aravena, M., et al. 2016, ApJ, 826, 112

Strandet, M. L., Weiss, A., De Breuck, C., et al. 2017, ApJL, 842, L15

Swinbank, A. M., Simpson, J. M., Smail, I., et al. 2014, MNRAS, 438, 1267

Tacconi, L. J., Genzel, R., Saintonge, A., et al. 2017, arXiv:1702.01140

Tacconi, L. J., Genzel, R., Smail, I., et al. 2008, ApJ, 680, 246

Tadaki, K.-I., Genzel, R., Kodama, T., et al. 2017a, ApJ, 834, 135

Tadaki, K.-I., Kodama, T., Nelson, E. J., et al. 2017b, ApJL, 841, L25

Ueda, J., Iono, D., Yun, M. S., et al. 2014, ApJS, 214, 1

Vieira, J. D., Marrone, D. P., Chapman, S. C., et al. 2013, Natur, 495, 344

Walter, F., Decarli, R., Aravena, M., et al. 2016, ApJ, 833, 67

Walter, F., Decarli, R., Carilli, C., et al. 2012, Natur, 486, 233

Wang, S. X., Brandt, W. N., Luo, B., et al. 2013, ApJ, 778, 179

Wardlow, J. L., Cooray, A., De Bernardis, F., et al. 2013, ApJ, 762, 59

Wardlow, J. L., Simpson, J. M., Smail, I., et al. 2018, MNRAS, 479, 3879

Weiß, A., Downes, D., Neri, R., et al. 2007, A\&A, 467, 955

Weiß, A., Kovács, A., Coppin, K., et al. 2009, ApJ, 707, 1201

Wuyts, S., Förster Schreiber, N. M., Wisnioski, E., et al. 2016, ApJ, 831, 149

Xue, Y. Q., Luo, B., Brandt, W. N., et al. 2011, ApJS, 195, 10 